\title{
Ethnobiological study on traditional medicinal plants and fungi recorded in the Naxi Dongba sutras
}

\author{
Haitao Li ${ }^{1,2}{ }^{\infty}$, Zhiyong $\mathrm{Li}^{3,4}$, Xiaobo Zhang ${ }^{5}$, Shaohua Yang ${ }^{6}$, Cui Chen ${ }^{6}$, Qingning Yang ${ }^{7}$, Chengfeng He ${ }^{7}$,
} Jianqin Liu ${ }^{7^{*}}$ and Jingyuan Song ${ }^{1 *}$

\begin{abstract}
Background: The Naxi people, living in Southwest China, have a long history and rich characteristic culture. Their ancestors recorded their life practices by ancient hieroglyphs and gradually formed the Dongba Sutras, which, among other knowledge, included the traditional knowledge of Naxi medicine. In the past, most studies on the Dongba Sutras focused on the humanistic culture of Naxi people, whereas studies have rarely focused on Naxi herbal medicinal plants and fungi described in the Dongba Sutras. Studying this aspect is helpful for exploring the traditional culture of Naxi people from the perspective of traditional medicine.
\end{abstract}

Methods: From February to September 2019, we screened the medicinal plants and fungi from the Dongba Sutras with the help of Dongba. Then, we carried out field investigations and collected voucher specimens of traditional medicinal plants and fungi with the help of 104 Naxi folk healers. The specimens were identified and stored in the Herbarium of Yunnan Branch, Institute of Medicinal Plants, Chinese Academy of Medical Sciences (IMDY). Through semi-structured interviews, we obtained ethnobotanical information of medicinal plants and fungi. The obtained quantitative data were analyzed using the informant consensus factor (ICF) method and the number of citations.

Results: A total of 85 species of medicinal plants and fungi belonging to 51 families and 71 genera were recorded in the Dongba Sutras. Among them, 25 species were endemic to China, and eight species were only distributed in Naxi distribution areas. These medicinal plants and fungi were mainly obtained from the wild, and 22 species could be used as food. The most frequent method of taking medicinal materials was oral-taking after decoction, followed by topical and sometimes buccal. The methods of processing these medicinal materials included water decoction, warm water flushing, and drinking after soaking. The medicinal plants and fungi in the Dongba Sutras are used to treat 96 conditions classified into 13 disease groups according to the International Classification of Primary Care second edition. Further analysis indicated that most of these species were utilized for treating diseases from the digestive (D) group, followed by those from the respiratory (R) group, musculoskeletal (L) group, general, and (Continued on next page)

\footnotetext{
* Correspondence: 2213964515@qq.com; jysong@implad.ac.cn

${ }^{7}$ Lijiang Medical Association of Minorities, Lijiang 674100, People's Republic of China

'Key Lab of Chinese Medicine Resources Conservation, State Administration of Traditional Chinese Medicine of the People's Republic of China, Institute of Medicinal Plant Development, Chinese Academy of Medical Sciences \& Peking Union Medical College, Beijing 100193, People's Republic of China Full list of author information is available at the end of the article
}

(c) The Author(s). 2021 Open Access This article is licensed under a Creative Commons Attribution 4.0 International License, which permits use, sharing, adaptation, distribution and reproduction in any medium or format, as long as you give appropriate credit to the original author(s) and the source, provide a link to the Creative Commons licence, and indicate if changes were made. The images or other third party material in this article are included in the article's Creative Commons licence, unless indicated otherwise in a credit line to the material. If material is not included in the article's Creative Commons licence and your intended use is not permitted by statutory regulation or exceeds the permitted use, you will need to obtain permission directly from the copyright holder. To view a copy of this licence, visit http://creativecommons.org/licenses/by/4.0/ The Creative Commons Public Domain Dedication waiver (http://creativecommons.org/publicdomain/zero/1.0/) applies to the data made available in this article, unless otherwise stated in a credit line to the data. 


\begin{abstract}
(Continued from previous page)
unspecified (A) group. Moreover, the Naxi people have a high consensus on the treatments of diseases from these four pathological groups.

Conclusions: The Naxi traditional medicine is characterized by simple materials, easy operation, and distinctive national characteristics. The ancient Naxi people recorded their highly developed medical culture in the Dongba Sutras. Natural plant resources found around them were their primary choices for both medicine and diet therapy. The ecological ethics of Naxi people have positive significance for the conservation of wild resources in their area.
\end{abstract}

Keywords: Ethnobiology, Lijiang city, The Dongba sutras, Naxi people, Traditional medicine

\section{Background}

The Naxi people inhabit areas of Southwest China, and they have a long history and a rich characteristic culture. Dongba symbols are the only hieroglyphs in the world that are still in use [1]. Joseph F. Rock collected about 8000 copies of Dongba scriptures, which were later deposited in major European and American libraries. Since his book was titled The Ancient Na-Khi Kingdom of Southwest China [2], Naxi people and their Dongba culture are famous throughout the world. The Dongba Sutras have become the main written materials for studying the Dongba culture. The Dongba Sutras is a special scripture and different from Buddhism Sutras or other classics. The content of Dongba Sutras covers the history, philosophy, society, religion, language and script, music, art, dance, and many other traditional subjects related to the Dongba culture. It is praised by academic circles as "the encyclopedia of ancient Naxi people" [3]. Naxi medical culture is an important part of Dongba culture. The Dongba Sutras contain information about the unique medical culture of the Naxi people, and they are the most important documents for studying Naxi medicine. The name "Dongba" is the appellation of the Naxi religious clergy and can be translated as "the wise." They are senior intellectuals and the main inheritors of the Dongba culture of the Naxi people, and most of them are skilled in singing, dancing, calligraphy, history, painting, and medicine.

Naxi ancestors have rich medical experience in the practice of fighting against diseases, and they created "Naxi medicine" or "Dongba medicine" [4]. These traditional medical experiences have been recorded by the Naxi people in the form of hieroglyphs, and they formed the Dongba Sutras. Only the Dongba who as the clergyman can recognize the hieroglyphics of Dongba sutras, and they lack of scientific research methods including ethnobotany. Therefore, in the existing literature, the medicinal plants and fungi recorded in Dongba sutras rarely corresponded to their scientific names.

Due to historical reasons, a large number of Dongba scriptures have been lost, some of them are scattered abroad or collected by privates. Currently, there are about 30,000 volumes of the Dongba Sutras, which are mainly stored in museums and libraries in China, the USA, Germany, France, Great Britain, and other countries [3]. These sutras are based on extensive experience in treating diseases and provide great knowledge of medicine. Chien Song Lü and Chongren Pandi to Find Medicine are the most representative sutras [5]. Chien Song L $\ddot{u}$ is the only medical book written in hieroglyphs of the Naxi people, and it includes data on dozens of medicinal plants. Chongren Pandi to Find Medicine includes records of the traditional treatment methods, the morphology, and function of some medicinal plants, and it has important reference value for the current medical practice [5]. The publication entitled The Complete Works of Dongba Sutras in Naxi [6] lays the foundation for deciphering the mysterious Naxi Dongba medicine.

In addition to the Dongba Sutras, in Naxi culture, a lot of valuable traditional knowledge has been transmitted orally, including a lot of precious medical information. Therefore, Naxi culture still needs to be further studied and systematically organized [4]. In recent decades, ethnomedicinal knowledge in Naxi communities has lost rapidly along with the high-speeded development of the Chinese economy. In particular, Lijiang is a famous tourism destination, and few young generations study traditional medicinal knowledge from the old generation. Less and less Naxi people use (or even recognize) traditional medicinal plants. Thus, it becomes very urgent and necessary to study medicinal plants recorded in the Dongba Sutras.

\section{Materials and methods}

\section{Study area}

Lijiang is a prefecture-level city in Yunnan Province, Southwest China. It is located in Hengduan Mountains, between $25^{\circ} 23^{\prime}-27^{\circ} 56^{\prime} \mathrm{N}$ and $99^{\circ} 23^{\prime}-101^{\circ} 11^{\prime} \mathrm{E}$. The total area of Lijiang City covers $20600 \mathrm{~km}^{2}$ [7]. The terrain of the area is high in the northwestern part and low in the southeastern part, with the highest altitude of $5596 \mathrm{~m}$ and the lowest altitude of $1015 \mathrm{~m}$. The maximum altitude difference of Lijiang is $4581 \mathrm{~m}$ [8].

The climate of Lijiang belongs to subtropical humid climate [9]. There is abundant rainfall and a distinct dry and wet season. The average annual rainfall is about 1 
$000 \mathrm{~mm}$, and the rainy season lasts from May to October being particularly pronounced in July and August. The annual average temperature is between $13{ }^{\circ} \mathrm{C}$ and $20{ }^{\circ} \mathrm{C}$, the average temperature of the hottest month is $18-26{ }^{\circ} \mathrm{C}$, and the average temperature of the coldest month is $4-12{ }^{\circ} \mathrm{C}$. Lijiang has $2500 \mathrm{~h}$ of annual sunshine and $147 \mathrm{kcal} / \mathrm{cm}^{2}$ of annual solar radiation [9].

Lijiang has a forest coverage rate of $70 \%$. The area is rich in medicinal materials and other exploitable biological resources and is known as the "kingdom of alpine plants" and "hometown of medicinal materials" [8].

The key areas of the present study were Gucheng District and Yulong County in Lijiang city, Yunnan Province, China. This area is the most concentrated area of the Naxi population in the world, with about 210000 people, accounting for $68.5 \%$ of the total Naxi population. Naxi people live in mountainous areas with inconvenient transportation and abundant biological resources, which is why their tradition is the most convenient mean of resisting diseases. At the same time, the inheritance model of Dongba culture is masters teaching apprentices that makes a better inheritance of the Naxi traditional medicinal culture.

\section{Data collection}

From February to September 2019, we screened the medicinal plants and fungi from the Dongba Sutras with the help of Dongba (the clergies who can read and write hieroglyphs) and translate the hieroglyphs into the Naxi language. Then, we carried out field research with assistance from 104 Naxi folk healers and collected traditional medicinal plant specimens. The basic survey information such as age and gender was collected and recorded. Using semi-structured interviews [10], ethnobotanical knowledge was obtained, including information about the local name, medicinal parts, harvesting methods, preparation methods, and indications of the medicinal plants and fungi from the Dongba Sutras. The informed consent of the participants was obtained before conducting the interviews, and the ethical guidelines prescribed by the International Society of Ethnobiology [11] were followed. The local names were transliterated from the Naxi or local Chinese pronunciation into the Roman alphabet following the Scheme for the Chinese Phonetic Alphabet [12] and the Basic Rules for Hanyu Pinyin Orthography [13]. The diseases treated by the medicinal plants and fungi from the Dongba Sutras were classified according to the International Classification of Primary Care (ICPC-2) [14] of the WHO (World Health Organization) $[15,16]$.

\section{Plant materials}

With the help of Naxi folk healers, 3-5 specimens of each species were collected, and the information about their habitats (e.g., altitude, latitude, longitude, and vegetation type), plant morphology (e.g., plant height, color of flowers, and corolla type), and date of the collection were recorded. The scientific and Chinese names were recorded on the label. These specimens were stored at the Herbarium, Yunnan Branch, Institute of Medicinal Plants, Chinese Academy of Medical Science (IMDY).

\section{Plant identification}

The following literature was used to identify the family and species names of the collected plants: Flora of China [17], Flora Reipublicae Popularis Sinicae [18], and Flora Yunnanica [19]. The scientific names were checked on The Plant List website [20]. All the plants listed are sorted at family level circumscription follows APG IV [21].

\section{Data analysis}

The data obtained in this study were analyzed using Microsoft Office Excel (2010) spreadsheet software. Quantitative data analysis was conducted using the informant consensus factor (ICF) method and the number of citations. ICF was calculated as ICF $=(\mathrm{Nur}-\mathrm{Nt}) /$ (Nur - 1), where Nur is the sum of plant species used by all the respondents to treat a particular disease, and $\mathrm{Nt}$ is the number of identical plant species used by all the respondents to treat a particular disease [22].

\section{Results and discussion}

\section{Demographic features of the respondents}

A total of 104 respondents were interviewed (Table 1). Among them, male respondents highly outnumbered the female respondents, and $79.81 \%$ of them were over 50 years old. Naxi people live in mountainous areas and commonly collect medicinal plants. In this harsh environment, men have an advantage over women due to their physical abilities. Because the experience of treating diseases is based on long-term practice, the medical experience mastered by older healers is more comprehensive and reliable than those learned by younger healers. Moreover, it ensures the reliability of the knowledge obtained in this survey.

The educational level of the respondents was generally low, and most of them had no higher education. However, this did not affect the reliability of the results, because the acquired traditional knowledge has truly maintained the characteristics of the Naxi people.

The respondents were mainly Naxi (71.158\%), followed by the Lisu (15.38\%). Other ethnic groups included Han, Tibetan, Bai, and Yi. All of these people lived in Naxi communities, and their medical skills were learned from Naxi healers. All the respondents were folk healers. Although there are many 
Table 1 Demographic features of the respondents

\begin{tabular}{|c|c|c|}
\hline Demographic features & Number & Proportion (\%) \\
\hline \multicolumn{3}{|l|}{ Age } \\
\hline $31-40$ & 7 & 6.73 \\
\hline $41-50$ & 14 & 13.46 \\
\hline $51-60$ & 28 & 26.92 \\
\hline $61-70$ & 24 & 23.08 \\
\hline $71-80$ & 24 & 23.08 \\
\hline 81 and above & 7 & 6.73 \\
\hline \multicolumn{3}{|l|}{ Sex } \\
\hline Female & 4 & 3.85 \\
\hline Male & 100 & 96.15 \\
\hline \multicolumn{3}{|l|}{ Education level } \\
\hline Illiterate & 10 & 9.62 \\
\hline Primary school & 61 & 58.65 \\
\hline Junior middle school & 12 & 11.54 \\
\hline Senior middle school & 8 & 7.69 \\
\hline Teacher training school & 1 & 0.96 \\
\hline School of health & 2 & 1.92 \\
\hline Polytechnic school & 5 & 4.81 \\
\hline Junior college & 4 & 3.85 \\
\hline University & 1 & 0.96 \\
\hline \multicolumn{3}{|l|}{ Nationality } \\
\hline Naxi & 74 & 71.15 \\
\hline Lisu & 16 & 15.38 \\
\hline Han & 5 & 4.81 \\
\hline Zang & 5 & 4.81 \\
\hline Bai & 3 & 2.88 \\
\hline Yi & 1 & 0.96 \\
\hline \multicolumn{3}{|l|}{ Ways of learning medicine } \\
\hline Ancestral & 73 & 70.19 \\
\hline Ancestral, ${ }^{*}$ master & 8 & 7.69 \\
\hline Master & 7 & 6.73 \\
\hline Ancestral, self-taught & 6 & 5.77 \\
\hline Master, self-taught & 4 & 3.85 \\
\hline Ancestral, learning at school & 2 & 1.92 \\
\hline Ancestral,self-taught, learning at school & 1 & 0.96 \\
\hline Master, learning at school & 1 & 0.96 \\
\hline Master,self-taught, learning at school & 1 & 0.96 \\
\hline Self-taught & 1 & 0.96 \\
\hline
\end{tabular}

*Master: an authority qualified to teach apprentices

ways to learn medical skills, most respondents (70.19\%) developed their medical experiences with the help of their ancestors. None of the respondents had regular jobs, and many of them were local Dongba who were priests and folk healers.
Table 2 Number of medicinal plants and fungi contained in the Dongba Sutras

\begin{tabular}{llll}
\hline Category & $\begin{array}{l}\text { Number of } \\
\text { families }\end{array}$ & $\begin{array}{l}\text { Number of } \\
\text { genera }\end{array}$ & $\begin{array}{l}\text { Number of } \\
\text { species }\end{array}$ \\
\hline Fungi & 3 & 3 & 3 \\
Pteridophyta & 3 & 3 & 3 \\
Gymnospermae & 2 & 3 & 5 \\
Angiospermae & 43 & 62 & 74 \\
Total & 51 & 71 & 85 \\
\hline
\end{tabular}

\section{Diversity of medicinal plants and fungi in the Dongba} sutras

According to our investigation, a total of 85 species of medicinal plants and fungi belonging to 51 families and 71 genera were recorded in the Dongba Sutras (Table 2). In the middle and high altitude areas, the main tree species belonged to the families Pinaceae, Cupressaceae, Ericaceae, and Fagaceae. Almost all parts of these plants can be used as medicine, especially their branches, which are often used by Naxi priests for various sacrificial activities. The highest numbers of plant species recorded belonged to the families Asteraceae (six species) and Polygonaceae (six species), followed by the Rosaceae (four species). It is worth mentioning that from the genus Rheum alone, we recorded three species. In addition to Rheum officinale recorded in the Pharmacopoeia of People's Republic of China [23], we also recorded $R$. delavayi and $R$. likiangense, but their usage was different from that of $R$. officinale recorded in the Pharmacopoeia of People's Republic of China.

Of all recorded species, herbaceous plants (49 species) accounted for the greatest number (Table 3), followed by trees ( 21 species) and shrubs ( 5 species). As herbaceous plants can more easily survive in a new environment than trees and shrubs [24], especially in the alpine mountains inhabited by the Naxi people, there is a lack of diversity of tree species, whereas the low herbaceous plants were abundant. At the same time, herbaceous plants are more convenient to collect than other plant

Table 3 Habits of medicinal plants and fungi contained in the Dongba Sutras

\begin{tabular}{|c|c|c|}
\hline Living habits & Number of species & Proportion (\%) \\
\hline Herbs & 46 & 54.12 \\
\hline Trees & 21 & 24.71 \\
\hline Shrubs & 5 & 5.88 \\
\hline Woody vines & 4 & 4.71 \\
\hline Climbing shrubs & 3 & 3.53 \\
\hline Herbaceous climbers & 3 & 3.53 \\
\hline Macro-fungi & 3 & 3.53 \\
\hline Total & 85 & 100.00 \\
\hline
\end{tabular}


life forms. Thus, the utilization rate of herbaceous plants is higher than that of trees and shrubs.

The medicinal parts of 85 medicinal plant and fungus species used by the respondents are indicated in Tables 4 and 10. The Naxi people knew that different medicinal parts have different effects. According to our analysis, in addition to the plant's medicinal efficacy, the difficulty of its collection also affects which parts would be used. The Naxi people preferred to collect easily collectible plant parts as raw materials for medicinal preparations. Among plant life forms, herbs and small shrubs are most commonly used as medicines, and the respondents reported that for this purpose, they used whole plants, roots, or rhizomes, whereas when trees, big shrubs, or woody vines are used for medicinal preparations, the respondents used stems, branches, leaves, or bark. The flowering and fruiting periods of these plants are short; therefore, their fruits, seeds, flowers, and buds are seldom used as medicinal parts. Plant secretions are rarely used as medicinal materials because of the difficulty of their collection.

Most of the medicinal plants in the Dongba Sutras are common plants in the studied area. The abundance of medicinal plants, determined according to the classification of abundance by Germany Ecologist Oscar Drude [25], is shown in Table 5. According to this classification, the highest number of species used by the respondents is forest species, such as Quercus aquifolioides, $Q$. aliena var. acuteserrata, Populus rotundifolia var. bonatii, and Pinus yunnanensis. The group with few or dispersed organism included only three species: Poria cocos, Dobinea delavayi, and Panax japonicus var. major. Although the medicinal materials from these species are

Table 4 Medicinal parts of plants and fungi recorded in the Dongba Sutras

\begin{tabular}{lll}
\hline Medicinal parts & Number of species & Proportion (\%) \\
\hline Roots or rhizomes & 29 & 24.37 \\
Whole plants & 22 & 18.49 \\
Leaves & 19 & 15.97 \\
Stems or branches & 17 & 14.29 \\
Fruits or seeds & 9 & 7.56 \\
Flowers or flower buds & 8 & 6.72 \\
Bark & 8 & 6.72 \\
Aerial parts & 3 & 2.52 \\
Fungi (fruit body) & 3 & 2.52 \\
Secretions & 1 & 0.84 \\
Total & 119 & 100.00 \\
\hline
\end{tabular}

Note: One or more parts of the same plant can be used as medicine, which is why the total number of medicinal parts exceeds the total number of species
Table 5 Abundance of medicinal plants and fungi contained in the Dongba Sutras

\begin{tabular}{lll}
\hline Abundance* & Number of species & Proportion (\%) \\
\hline Soe & 4 & 4.71 \\
Cop3 & 13 & 15.29 \\
Cop2 & 17 & 20.00 \\
Cop1 & 38 & 44.71 \\
Sp & 10 & 11.76 \\
Sol & 3 & 3.53 \\
Un & 0 & 0.00 \\
Total & 85 & 100.00
\end{tabular}

*Soe (Sociales): High number of individuals, the above-ground plant part is closed

Cop3 (Copiosae): High number of individuals, but the above-ground plant part is not closed

Cop2: Large and common plants

Cop1: Large plants, but small populations

Sp (Sparsal): Low number of plants, scattered

Sol (Solitariae): Low number of plants, sparse

Un (Unicum): Only one individual

rarely found in the wild, they have been cultivated in the area and thus have been successfully used as medicines.

Since ancient times, Naxi people have lived in mountainous areas, where transportation is inconvenient. The medicines they used were collected in the mountains, and rare medicinal plants were cultivated in their courtyards in order to be convenient for collection. Therefore, the medicinal plants described in the Dongba Sutras were mainly wild plants, accounting for $76.47 \%$ of all medicinal plants described in the Dongba Sutras (Table 6). Because of the small population of Naxi people, their use of wild medicinal plants does not present a threat to the stability of wild plant populations.

Food therapy is an important characteristic of Chinese culture and traditional Chinese medicine (TCM). "One Root of medicine and food" is a summary of the Chinese people's understanding of medicine and food and their relationship [26]. The life of the Naxi people is closely related with medical dietary plants, and their medicinal diets are indispensable to the health of their communities [27]. Among the medicinal plants in the Dongba Sutras, 22 species can be consumed as vegetables, fruits, dried fruits, or condiments (Table 7). For example, Lagenaria siceraria, Brassica rapa, Foeniculum vulgare, and Allium ascalonicum are common vegetable species. Setaria italica

Table 6 Sources of drugs contained in the Dongba Sutras

\begin{tabular}{lll}
\hline Sources & Number of species & Proportion (\%) \\
\hline Wild & 65 & 76.47 \\
Cultivated & 7 & 8.24 \\
Mixture of wild and cultivated & 13 & 15.29 \\
Total & 85 & 100.00 \\
\hline
\end{tabular}


Table 7 List of medicinal and edible plants and fungi recorded in the Dongba Sutras

\begin{tabular}{|c|c|c|c|c|}
\hline ID & Family & Scientific name & Resource type & Food type \\
\hline 1 & Amaranthaceae & Amaranthus hypochondriacus & Wild & Vegetable \\
\hline 2 & Amaryllidaceae & Allium ascalonicum & Cultivated & Vegetable \\
\hline 3 & Amaryllidaceae & Allium hookeri & Cultivated, wild & Vegetable \\
\hline 4 & Amaryllidaceae & Allium sativum & Cultivated & Vegetable \\
\hline 5 & Apiaceae & Foeniculum vulgare & Cultivated & Vegetable \\
\hline 6 & Brassicaceae & Brassica rapa & Cultivated & Vegetable \\
\hline 7 & Cactaceae & Opuntia ficus-indica & Cultivated, wild & Fruit \\
\hline 8 & Cannabaceae & Cannabis sativa & Cultivated, wild & Condiment \\
\hline 9 & Cucurbitaceae & Lagenaria siceraria & Cultivated & Vegetable \\
\hline 10 & Ebenaceae & Diospyros lotus & Cultivated, wild & Fruit \\
\hline 11 & Fabaceae & Pueraria montana var. chinensis & Wild & Beverage \\
\hline 12 & Juglandaceae & Juglans regia & Cultivated, wild & Dry fruit \\
\hline 13 & Lamiaceae & Elsholtzia rugulosa & Wild & Beverage \\
\hline 14 & Lauraceae & Neocinnamomum delavayi & Wild & Condiment \\
\hline 15 & Pinaceae & Pinus armandii & Wild & Dry fruit \\
\hline 16 & Poaceae & Setaria italica var. germanica & Cultivated & Food \\
\hline 17 & Polyporaceae & Poria cocos & Wild & Vegetable \\
\hline 18 & Rosaceae & Prunus mume & Cultivated, wild & Fruit \\
\hline 19 & Rosaceae & Rubus biflorus & Wild & Fruit \\
\hline 20 & Rosaceae & Rubus coreanus var. tomentosus & Wild & Fruit \\
\hline 21 & Rosaceae & Rubus niveus & Wild & Fruit \\
\hline 22 & Schizophyllaceae & Schizophyllum commune & Wild & Vegetable \\
\hline
\end{tabular}

var. germanica is also used as food. For a long time, the Naxi people considered that these foods and vegetables can be used to treat and prevent diseases. Thus, they recorded them in the Dongba Sutras. Some of these medicines are used to prepare tea and do not have any negative side effects. For example, the aerial parts of Elsholtzia rugulosa which has the effect of relieving summer heat. The plant as a substitute for tea is easy to collect and prepare and has widely been used by the Naxi people. This indicated that in the Naxi people, maintaining a healthy daily diet is a very important factor in disease prevention.

\section{Medicine preparation methods and applications}

The folk preparation methods of traditional Naxi medicine were relatively simple (Fig. 1a); most of them included washing and direct drying of the plant material (49.18\%), followed by crushing (20.49\%), soaking (13.93\%), using fresh products $(9.84 \%)$, blending with other agents (3.28\%), and carbonization (3.28\%). The medium used in the soaking process was mainly wine or water, whereas the medium used in blending included edible oils, vinegar, and honey, etc. The use of fresh plant parts as medicine is characteristic of Naxi medicine because this method is simpler to use than other methods. In this method, the medicinal parts are removed from the plants and washed, and they are used after mashing or chewing. In addition, juice extracted directly from the plant is also a common method of fresh plant intake and is mostly used for topical application. The main method of medicine consummation was oral, followed by topical and rarely buccal (Fig. 1b). Oral administration included three methods: boiling in water, washing in warm water, and drinking after soaking.

\section{ICF, conditions, and diseases treated by the studied plants and fungi}

The informant consensus factor (ICF) is a measure of information diversity. The higher the ICF value, the greater the difference among plant species used in the treatment of a given disease, and the lower the ICF value, the smaller the difference among plant species used in the treatment of a disease [22]. We found that the medicinal plants and fungi in the Dongba Sutras are used to treat 96 conditions, which can be classified into 13 disease groups according to ICPC-2 (Table 8 and Table 9). The highest ICF values were recorded for the eye group (F), cardiovascular group (K), and psychological group $(\mathrm{P})(\mathrm{ICF}=1.50)$, followed by the neurological group $(\mathrm{N})$, female genital group $(\mathrm{X})$, and male 


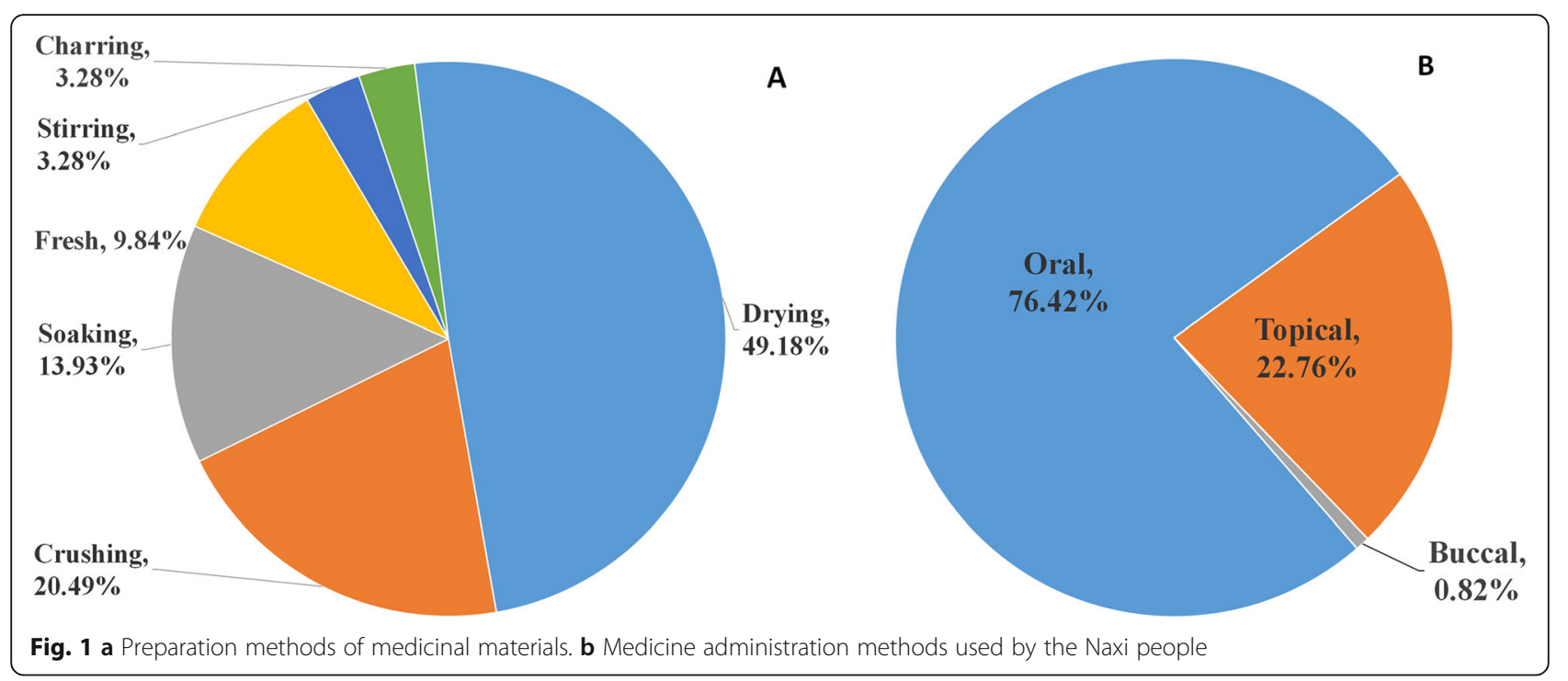

genital group (Y) (ICF: 1.00). Among the medicinal plants provided by different respondents, there are very few (only one or none) identical plants that can be used to treat the same group of diseases. This showed that there are many differences among Naxi people in the methods of treating a specific disease, i.e., that they have low consensus about disease treatment methods. There are two possible reasons for this: (1) as the Naxi people live in biodiversity-rich areas, the abundant medicinal plant resources provided them with a wide choice of medicinal plants to use [28], and (2) different Naxi folk healers may have different degrees of understanding of

Table 8 Informant consensus factor (ICF) values of the medicinal plants and fungi contained in the Dongba Sutras

\begin{tabular}{llll}
\hline Disease types & $\begin{array}{l}\text { The sum of plant } \\
\text { species (Nur) }\end{array}$ & $\begin{array}{l}\text { The number of } \\
\text { identical plant } \\
\text { species used (Nt) }\end{array}$ & ICF \\
\hline $\begin{array}{l}\text { A: General and } \\
\text { unspecified }\end{array}$ & 21 & 6 & 0.75 \\
D: Digestive & 36 & 15 & 0.60 \\
F: Eye & 3 & 0 & 1.50 \\
K: Cardiovascular & 3 & 0 & 1.50 \\
L: Musculoskeletal & 21 & 12 & 0.45 \\
N: Neurological & 4 & 1 & 1.00 \\
P: Psychological & 3 & 0 & 1.50 \\
R: Respiratory & 29 & 13 & 0.57 \\
S: Skin & 11 & 2 & 0.90 \\
T: Endocrine/metabolic & 1 & 1 & - \\
and nutritional & & 4 & 0.73 \\
U: Urological & 12 & 1 & 1.00 \\
X: Female genital & 16 & 1 & 1.00 \\
Y: Male genital & 14 & &
\end{tabular}

the same disease (e.g., some may be focused more on the symptoms of a disease, but ignore or miss the real cause of the disease).

Further analysis indicated that most of the plant species were utilized for the group of digestive diseases (D; Nur $=36, \mathrm{Nt}=15)$, followed by the respiratory $(\mathrm{R} ; \mathrm{Nur}=29$, $\mathrm{Nt}=13$,), musculoskeletal ( $\mathrm{L}$; Nur=21, $\mathrm{Nt}=12$ ), and the general and unspecified disease group $(\mathrm{A} ; \mathrm{Nur}=21, \mathrm{Nt}=$ $5)$. The ICF values of these four disease groups were low: Group D: 0.60, Group R: 0.57, group L: 0.45, and group A: 0.75 . These low values indicated that these four groups of diseases are common diseases in Naxi people living areas, and Naxi folk healers have a high consensus on the treatment of these diseases.

For the treatment of diabetes (T89: Diabetes Insulin Dependent or T90: Diabetes Non-Insulin Dependent), which is an endocrine disease that belongs to the group of endocrine/metabolic and nutritional, only one plant species was cited in the Dongba Sutras. Diaphragma juglandis fructus, the dry wood diaphragm tissue (xylem septa) that grows inside the walnut (Juglans regia), was reported as a medicinal plant that can be used to treat diabetes, and the consensus on this treatment was high. A previous study reported that the flavonoids from Diaphragma juglandis fructus have significant anti-diabetic activity [29]. This shows that as the knowledge on folk medicine is collected from long-term practical experience, its scientific nature has yet to be proven by modern science. With more research, more information from traditional medicinal practices will be scientifically proven.

The plant species with the highest number of use reports were Rheum likiangense (13 use reports), Reineckea carnea (11 use reports), Rheum delavayi (10 use reports), and Hypericum augustinii (10 use reports). Rheum likiangense and $R$. delavayi are endemic to a 
Table 9 Ethnomedicinal data of medicinal plants and fungi recorded in the Dongba Sutras

\begin{tabular}{|c|c|c|c|c|c|c|c|}
\hline $\begin{array}{l}\text { Chinese } \\
\text { name }\end{array}$ & $\begin{array}{l}\text { Naxi } \\
\text { name }\end{array}$ & Scientific name & $\begin{array}{l}\text { Family/voucher } \\
\text { specimen/ } \\
\text { habitat }^{\mathrm{a}} / \text { habit }^{\mathrm{b}}\end{array}$ & Part used & $\begin{array}{l}\text { Preparation } \\
\text { method }\end{array}$ & $\begin{array}{l}\text { Route of } \\
\text { administration }\end{array}$ & $\begin{array}{l}\text { Diseases treated/number of } \\
\text { respondents (ICPC-2) }\end{array}$ \\
\hline $\begin{array}{l}\text { Zhu Sheng } \\
\text { Rou Qiu } \\
\text { Jun }\end{array}$ & Men Mu & $\begin{array}{l}\text { Engleromyces goetzi P. } \\
\text { Henn. }\end{array}$ & $\begin{array}{l}\text { Hypocreaceae/ } \\
\text { NX0759/W/M }\end{array}$ & Fruit body & Drying & Oral & $\begin{array}{l}\text { Elevated Blood Pressure K85 } \\
\text { (57)Headache N01 (64) } \\
\text { Throat Symptom R21 (52) }\end{array}$ \\
\hline $\begin{array}{l}\text { Lie Zhe } \\
\text { Jun }\end{array}$ & $\begin{array}{l}\text { Si Du } \\
\text { Mou Pei }\end{array}$ & $\begin{array}{l}\text { Schizophyllum commune } \\
\text { Franch. }\end{array}$ & $\begin{array}{l}\text { Schizophyllaceae/ } \\
\text { NX0360/W/M }\end{array}$ & Fruit body & Drying & Oral & $\begin{array}{l}\text { Cough R05 (104) } \\
\text { Pleurisy/Pleural Effusion R82 } \\
\text { (104) }\end{array}$ \\
\hline Fu Ling & $\begin{array}{l}\text { Tuo Ken } \\
\text { Liu }\end{array}$ & Poria cocos (Schw.) Wolf & $\begin{array}{l}\text { Polyporaceae/ } \\
\text { NX0581/W/M }\end{array}$ & Fruit body & $\begin{array}{l}\text { Drying/ } \\
\text { Soaking }\end{array}$ & Oral/Topical & $\begin{array}{l}\text { Gonorrhoea Female X71 (12) } \\
\text { Limited Function/Disability (L) } \\
\text { L28 (68) }\end{array}$ \\
\hline $\begin{array}{l}\text { Dian } \\
\text { Zhuang } \\
\text { Juan Bai }\end{array}$ & $\begin{array}{l}\text { Ci Liu } \\
\text { Liu Ru } \\
\text { Da Bie }\end{array}$ & $\begin{array}{l}\text { Selaginella pulvinata } \\
\text { (Hook. et Grev.) Maxim. }\end{array}$ & $\begin{array}{l}\text { Selaginellaceae/ } \\
\text { NX0281/W/H }\end{array}$ & Roots & Charring & Oral & $\begin{array}{l}\text { Bleeding/Hemorrhage NOS A10 } \\
\text { (76) }\end{array}$ \\
\hline Jie Jie Cao & $\begin{array}{l}\text { Mie Liu } \\
\text { Ku Sa }\end{array}$ & $\begin{array}{l}\text { Equisetum ramosissimum } \\
\text { Desf. }\end{array}$ & $\begin{array}{l}\text { Equisetaceae/ } \\
\text { NX0364, NX0657/ } \\
\text { W/H }\end{array}$ & $\begin{array}{l}\text { Whole } \\
\text { plants }\end{array}$ & $\begin{array}{l}\text { Charring/ } \\
\text { Drying }\end{array}$ & Oral & $\begin{array}{l}\text { Eye Discharge F03 (80) } \\
\text { Genital Disease Male other Y99 } \\
\text { (46) } \\
\text { Menstruation Excessive X06 (72) }\end{array}$ \\
\hline $\begin{array}{l}\text { Chuan } \\
\text { Dian Hu } \\
\text { Jue }\end{array}$ & $\begin{array}{l}\text { Lu Ba Di } \\
\text { Li }\end{array}$ & Drynaria delavayi Christ & $\begin{array}{l}\text { Drynariaceae/ } \\
\text { NX0151/W/H }\end{array}$ & Rhizomes & Crushing & Topical & $\begin{array}{l}\text { Fracture: Femur L75 (78) } \\
\text { Fracture: Hand / Foot Bone L74 } \\
\text { (64) } \\
\text { Fracture: Other L76 (43) } \\
\text { Fracture: Radius/Ulna L72 (37) } \\
\text { Fracture: Tibia/Fibula L73 (90) } \\
\text { Limited Function/Disability (L) } \\
\text { L28 (89) } \\
\text { Musculoskeletal Disease other } \\
\text { L99 (44) } \\
\text { Osteoarthrosis other L91 (37) } \\
\text { Pelvis Symptom/Complaint } \\
\text { Female X17 (18) }\end{array}$ \\
\hline $\begin{array}{l}\text { Li Jiang } \\
\text { Yun Shan }\end{array}$ & $\begin{array}{l}\text { Li Ben } \\
\text { Le }\end{array}$ & $\begin{array}{l}\text { Picea likiangensis } \\
\text { (Franch.) Pritz }\end{array}$ & $\begin{array}{l}\text { Pinaceae/NX0318/ } \\
\text { W/T }\end{array}$ & Fruits & Drying & Oral & $\begin{array}{l}\text { Osteoarthrosis other L91 (102) } \\
\text { Rheumatoid/Seropositive } \\
\text { Arthritis L88 (99) }\end{array}$ \\
\hline $\begin{array}{l}\text { Hua Shan } \\
\text { Song }\end{array}$ & Se Tong & Pinus armandii Franch. & $\begin{array}{l}\text { Pinaceae/NX0223, } \\
\text { NX0322/W/T }\end{array}$ & Secretion & Drying & Oral & $\begin{array}{l}\text { Constipation D12 (25) } \\
\text { Cough R05 (52) } \\
\text { Epilepsy N88 (37) }\end{array}$ \\
\hline $\begin{array}{l}\text { Yun Nan } \\
\text { Song }\end{array}$ & $\begin{array}{l}\mathrm{Ge} \mathrm{Bo} \\
\mathrm{Ha}\end{array}$ & $\begin{array}{l}\text { Pinus yunnanensis } \\
\text { Franch. }\end{array}$ & $\begin{array}{l}\text { Pinaceae/NX0159/ } \\
\text { W/T }\end{array}$ & $\begin{array}{l}\text { Flowers, } \\
\text { branches }\end{array}$ & $\begin{array}{l}\text { Crushing/ } \\
\text { stirring }\end{array}$ & Oral & $\begin{array}{l}\text { Acute Bronchitis/Bronchiolitis } \\
\text { R78 (75) } \\
\text { Chronic Bronchitis R79 (84) } \\
\text { Limited Function/Disability (L) } \\
\text { L28 (28) } \\
\text { Pneumonia R81 (76) } \\
\text { Tuberculosis A70 (23) }\end{array}$ \\
\hline $\begin{array}{l}\text { Gan Xiang } \\
\text { Bai }\end{array}$ & $\begin{array}{l}\text { Xiong } \\
\text { Ban }\end{array}$ & $\begin{array}{l}\text { Cupressus duclouxiana } \\
\text { Hickel }\end{array}$ & $\begin{array}{l}\text { Cupressaceae/ } \\
\text { NX0558/W/T }\end{array}$ & $\begin{array}{l}\text { Branches, } \\
\text { leaves }\end{array}$ & Drying & Topical & $\begin{array}{l}\text { Leg/Thigh Symptom L14 (87) } \\
\text { Low Back Symptom L03 (68) } \\
\text { Muscle Pain L18 (104) }\end{array}$ \\
\hline $\begin{array}{l}\text { Gao Shan } \\
\text { Bai }\end{array}$ & Xiu Xu & $\begin{array}{l}\text { Juniperus squamata } \\
\text { Buch.-Ham. ex D.Don }\end{array}$ & $\begin{array}{l}\text { Cupressaceae/ } \\
\text { NX0257, NX0614/ } \\
\text { W/S or T }\end{array}$ & $\begin{array}{l}\text { Branches, } \\
\text { leaves }\end{array}$ & Drying & Oral/Topical & $\begin{array}{l}\text { Intermenstrual Bleeding X08 } \\
\text { (100) } \\
\text { Menstruation Irregular/Frequent } \\
\text { X07 (86) }\end{array}$ \\
\hline $\begin{array}{l}\text { Hong Hua } \\
\text { Wu Wei Zi }\end{array}$ & $\begin{array}{l}\text { Gua Ji } \\
\text { Liu }\end{array}$ & $\begin{array}{l}\text { Schisandra rubriflora } \\
\text { Rehder et E.H.Wilson }\end{array}$ & $\begin{array}{l}\text { Schisandraceae/ } \\
\text { NX0248/W/WV }\end{array}$ & Bark & Soaking & Oral & $\begin{array}{l}\text { Abdominal Pain Localized other } \\
\text { D06 (44) } \\
\text { Cystitis/Urinary Infection other } \\
\text { U71 (32) } \\
\text { Pain General/Multiple Sites A01 } \\
\text { (104) } \\
\text { Sleep Disturbance P06 (46) } \\
\text { Trauma/Injury A80 (104) }\end{array}$ \\
\hline $\begin{array}{l}\text { Shan Yu } \\
\text { Lan }\end{array}$ & $\begin{array}{l}\text { Han Yi } \\
\text { Ba Da }\end{array}$ & $\begin{array}{l}\text { Magnolia delavayi } \\
\text { Franch. }\end{array}$ & $\begin{array}{l}\text { Magnoliaceae/ } \\
\text { NX0701/C/T }\end{array}$ & Flowers & Soaking & Oral & $\begin{array}{l}\text { Abdominal Pain Epigastric D02 } \\
\text { (53) }\end{array}$ \\
\hline
\end{tabular}


Table 9 Ethnomedicinal data of medicinal plants and fungi recorded in the Dongba Sutras (Continued)

\begin{tabular}{|c|c|c|c|c|c|c|c|}
\hline $\begin{array}{l}\text { Chinese } \\
\text { name }\end{array}$ & $\begin{array}{l}\text { Naxi } \\
\text { name }\end{array}$ & Scientific name & $\begin{array}{l}\text { Family/voucher } \\
\text { specimen/ } \\
\text { habitat }^{\mathrm{a}} / \text { habit }^{\mathrm{b}}\end{array}$ & Part used & $\begin{array}{l}\text { Preparation } \\
\text { method }\end{array}$ & $\begin{array}{l}\text { Route of } \\
\text { administration }\end{array}$ & $\begin{array}{l}\text { Diseases treated/number of } \\
\text { respondents (ICPC-2) }\end{array}$ \\
\hline Xin Zhang & Sei Bi & $\begin{array}{l}\text { Neocinnamomum } \\
\text { delavayi (Lec.)H. Liu }\end{array}$ & $\begin{array}{l}\text { Lauraceae/ } \\
\text { NX0760/W/T }\end{array}$ & $\begin{array}{l}\text { Leaves, } \\
\text { bark }\end{array}$ & Stirring & Topical & Diarrhoea D11 (45) \\
\hline Chang Pu & $\begin{array}{l}\text { Ji Chu } \\
\text { Buer }\end{array}$ & Acorus calamus Linn. & $\begin{array}{l}\text { Acoraceae/ } \\
\text { NX0116/C, W/H }\end{array}$ & $\begin{array}{l}\text { Whole } \\
\text { plants }\end{array}$ & Crushing & Oral & $\begin{array}{l}\text { Abdominal Pain Epigastric D02 } \\
\text { (99) } \\
\text { Influenza R80 (104) } \\
\text { Mumps D71 (104) } \\
\text { Upper Respiratory Infection } \\
\text { Acute R74 (58) }\end{array}$ \\
\hline $\begin{array}{l}\text { Dong } \\
\text { Fang Ze } \\
\text { Xie }\end{array}$ & $\begin{array}{l}\mathrm{He} \mathrm{Ke} \\
\mathrm{Gu}\end{array}$ & $\begin{array}{l}\text { Alisma orientale (Samuel.) } \\
\text { Juz. }\end{array}$ & $\begin{array}{l}\text { Alismataceae/ } \\
\text { NX0520/C,W/H }\end{array}$ & Roots & Drying & Oral & $\begin{array}{l}\text { Dysuria/Painful Urination U01 } \\
\text { (72) }\end{array}$ \\
\hline Shou Shen & $\begin{array}{l}\text { A You } \\
\mathrm{La} \mathrm{Ba}\end{array}$ & $\begin{array}{l}\text { Gymnadenia conopsea } \\
\text { (Linn.) R. Br. }\end{array}$ & $\begin{array}{l}\text { Orchidaceae/ } \\
\text { NX0352/W/H }\end{array}$ & Roots & $\begin{array}{l}\text { Crushing/ } \\
\text { drying }\end{array}$ & Oral & $\begin{array}{l}\text { Cough R05 (74) } \\
\text { Low Back Symptom L03 (45) } \\
\text { Pain General/Multiple Sites A01 } \\
\text { (55) } \\
\text { Sexual Function Symptom/ } \\
\text { Complaint Male Y08 (31) }\end{array}$ \\
\hline $\begin{array}{l}\text { Xi Nan } \\
\text { Shou Shen }\end{array}$ & $\begin{array}{l}\text { A You } \\
\text { La Ba }\end{array}$ & $\begin{array}{l}\text { Gymnadenia orchidis } \\
\text { Lindl. }\end{array}$ & $\begin{array}{l}\text { Orchidaceae/ } \\
\text { NX0349/W/H }\end{array}$ & Roots & $\begin{array}{l}\text { Crushing/ } \\
\text { drying }\end{array}$ & Oral & $\begin{array}{l}\text { Cough R05 (74) } \\
\text { Low Back Symptom L03 (45) } \\
\text { Pain General/Multiple Sites A01 } \\
\text { (55) } \\
\text { Sexual Function Symptom / } \\
\text { Complaint Male Y08 (31) }\end{array}$ \\
\hline Shou Cao & $\begin{array}{l}\mathrm{Lu} \mathrm{Bu} \\
\mathrm{Ge}\end{array}$ & $\begin{array}{l}\text { Spiranthes sinensis (Pers.) } \\
\text { Ames }\end{array}$ & $\begin{array}{l}\text { Orchidaceae/ } \\
\text { NX0122, NX0544/ } \\
\text { W/H }\end{array}$ & $\begin{array}{l}\text { Whole } \\
\text { plants }\end{array}$ & $\begin{array}{l}\text { Crushing/ } \\
\text { soaking/ } \\
\text { stirring }\end{array}$ & Oral/Topical & $\begin{array}{l}\text { Herpes Zoster S70 (53) } \\
\text { Low Back Symptom L03 (86) } \\
\text { Pain General/Multiple Sites A01 } \\
\text { (97) } \\
\text { Sexual Function Symptom/ } \\
\text { Complaint Male Y08 (24) }\end{array}$ \\
\hline Huo Cong & $\begin{array}{l}\text { Cong Ke } \\
\text { Pei Er }\end{array}$ & Allium ascalonicum Linn. & $\begin{array}{l}\text { Amaryllidaceae/ } \\
\text { NX0746/C/H }\end{array}$ & $\begin{array}{l}\text { Whole } \\
\text { plants }\end{array}$ & Drying & Oral & $\begin{array}{l}\text { Influenza R80 (69) } \\
\text { Upper Respiratory Infection } \\
\text { Acute R74 (104) }\end{array}$ \\
\hline $\begin{array}{l}\text { Kuan Ye } \\
\text { Jiu }\end{array}$ & $\begin{array}{l}\text { Ju Ge } \\
\text { Shu }\end{array}$ & Allium hookeri Thwaites & $\begin{array}{l}\text { Amaryllidaceae/ } \\
\text { NX0705/C, W/H }\end{array}$ & $\begin{array}{l}\text { Leaves, } \\
\text { roots }\end{array}$ & Fresh & Topical & Allergy/Allergic Reation A92 (93) \\
\hline Suan & $\mathrm{Gu}$ & Allium sativum Linn. & $\begin{array}{l}\text { Amaryllidaceae/ } \\
\text { NX0764/C/H }\end{array}$ & $\begin{array}{l}\text { Whole } \\
\text { plants }\end{array}$ & Drying/fresh & Oral/Topical & $\begin{array}{l}\text { Animal/Human Bite S13 (98) } \\
\text { Insect Bite/Sting S12 (104) }\end{array}$ \\
\hline $\begin{array}{l}\text { Mi Chi } \\
\text { Tian Men } \\
\text { Dong }\end{array}$ & $\mathrm{Ai} X u$ & $\begin{array}{l}\text { Asparagus meioclados } \\
\text { Lévl. }\end{array}$ & $\begin{array}{l}\text { Asparagaceae/ } \\
\text { NX0640/W/H }\end{array}$ & Roots & Drying & Oral & $\begin{array}{l}\text { Cough R05 (104) } \\
\text { Respiratory Disease other R99 } \\
(69)\end{array}$ \\
\hline $\begin{array}{l}\text { Ji Xiang } \\
\text { Cao }\end{array}$ & $\begin{array}{l}\text { Gu Ke } \\
\text { Gu Zhe } \\
\text { Le }\end{array}$ & $\begin{array}{l}\text { Reineckea carnea (Andr.) } \\
\text { Kunth }\end{array}$ & $\begin{array}{l}\text { Asparagaceae/ } \\
\text { NX0651/W/H }\end{array}$ & $\begin{array}{l}\text { Whole } \\
\text { plants }\end{array}$ & $\begin{array}{l}\text { Crushing/ } \\
\text { fresh/drying }\end{array}$ & Oral/Topical & $\begin{array}{l}\text { Acute Bronchitis/Bronchiolitis } \\
\text { R78 (101) } \\
\text { Chronic Bronchitis R79 (103) } \\
\text { Cystitis/Urinary Infection other } \\
\text { U71 (45) } \\
\text { Fracture: Femur L75 (79) } \\
\text { Fracture: Hand/Foot Bone L74 } \\
\text { (65) } \\
\text { Fracture: Other L76 (47) } \\
\text { Fracture: Radius/Ulna L72 (53) } \\
\text { Fracture: Tibia/Fibula L73 (100) } \\
\text { Genital symptom/Complaint } \\
\text { Female other X29 (36) } \\
\text { Low Back Symptom L03 (100) } \\
\text { Pain General/Multiple Sites A01 } \\
\text { (66) }\end{array}$ \\
\hline $\begin{array}{l}\text { Dian Jiang } \\
\text { Hua }\end{array}$ & Gu Shu & $\begin{array}{l}\text { Hedychium yunnanense } \\
\text { Gagnep. }\end{array}$ & $\begin{array}{l}\text { Zingiberaceae/ } \\
\text { NX0610/W/H }\end{array}$ & Roots & Drying/fresh & Oral/Topical & $\begin{array}{l}\text { Influenza R80 (88) } \\
\text { Orchitis/Epididymitis Y74 (18) } \\
\text { Osteoarthrosis Other L91 (64) } \\
\text { Rheumatoid/Seropositive } \\
\text { Arthritis L88 (59) }\end{array}$ \\
\hline
\end{tabular}


Table 9 Ethnomedicinal data of medicinal plants and fungi recorded in the Dongba Sutras (Continued)

\begin{tabular}{|c|c|c|c|c|c|c|c|}
\hline $\begin{array}{l}\text { Chinese } \\
\text { name }\end{array}$ & $\begin{array}{l}\text { Naxi } \\
\text { name }\end{array}$ & Scientific name & $\begin{array}{l}\text { Family/voucher } \\
\text { specimen/ } \\
\text { habitat }^{\mathrm{a}} / \text { habit }^{\mathrm{b}}\end{array}$ & Part used & $\begin{array}{l}\text { Preparation } \\
\text { method }\end{array}$ & $\begin{array}{l}\text { Route of } \\
\text { administration }\end{array}$ & $\begin{array}{l}\text { Diseases treated/number of } \\
\text { respondents (ICPC-2) }\end{array}$ \\
\hline & & & & & & & $\begin{array}{l}\text { Upper Respiratory Infection } \\
\text { Acute R74 (74) }\end{array}$ \\
\hline $\begin{array}{l}\text { Chang } \\
\text { Yuan Qiao } \\
\text { Jian Zhu }\end{array}$ & Ju Me & $\begin{array}{l}\text { Fargesia orbiculata T. P. } \\
\text { Yi }\end{array}$ & $\begin{array}{l}\text { Poaceae/NX0665/ } \\
\text { W/S }\end{array}$ & Leaves & Charring & Oral & $\begin{array}{l}\text { Influenza R80 (58) } \\
\text { Trauma/Injury A80 (28) } \\
\text { Upper Respiratory Infection } \\
\text { Acute R74 (79) }\end{array}$ \\
\hline Su & $\begin{array}{l}\text { Chong } \\
\text { Jing }\end{array}$ & $\begin{array}{l}\text { Setaria italica var. } \\
\text { germanica (Mill.) Schred. }\end{array}$ & $\begin{array}{l}\text { Poaceae/NX0765/ } \\
\text { C/H }\end{array}$ & $\begin{array}{l}\text { Whole } \\
\text { plants }\end{array}$ & Drying & Oral & Dyspepsia/Indigestion D07 (35) \\
\hline $\begin{array}{l}\text { Jin Mao } \\
\text { Tie Xian } \\
\text { Lian }\end{array}$ & $\begin{array}{l}\text { Hai Ke Si } \\
\text { Zi Beng }\end{array}$ & $\begin{array}{l}\text { Clematis chrysocoma } \\
\text { Franch. }\end{array}$ & $\begin{array}{l}\text { Ranunculaceae/ } \\
\text { NX0370/W/WV }\end{array}$ & $\begin{array}{l}\text { Whole } \\
\text { plants }\end{array}$ & Drying & Oral & Bladder Symptom U13 (67) \\
\hline $\begin{array}{l}\text { He Bing } \\
\text { Tie Xian } \\
\text { Lian }\end{array}$ & $\begin{array}{l}\text { Ze Die } \\
\text { Ba }\end{array}$ & Clematis connata DC. & $\begin{array}{l}\text { Ranunculaceae/ } \\
\text { NX0721/W/WV }\end{array}$ & Stem & Drying & Oral & $\begin{array}{l}\text { Pelvis Symptom/Complaint } \\
\text { Female other X17 (39) }\end{array}$ \\
\hline $\begin{array}{l}\text { Pao Hua } \\
\text { Shu }\end{array}$ & $\begin{array}{l}\text { Gai Si } \\
\text { Ze }\end{array}$ & $\begin{array}{l}\text { Meliosma cuneifolia } \\
\text { Franch. }\end{array}$ & $\begin{array}{l}\text { Sabiaceae/ } \\
\text { NX0669/W/T }\end{array}$ & $\begin{array}{l}\text { Leaves, } \\
\text { stem }\end{array}$ & Drying & Oral & $\begin{array}{l}\text { Cystitis/Urinary Infection other } \\
\text { U71 (15) }\end{array}$ \\
\hline $\begin{array}{l}\text { Chuan } \\
\text { Dian Que } \\
\text { Er Dou }\end{array}$ & $\begin{array}{l}\text { Wen Lu } \\
\text { Ban Qi } \\
\text { Shi }\end{array}$ & $\begin{array}{l}\text { Chesneya polystichoides } \\
\text { (Hand.-Mazz.) Ali }\end{array}$ & $\begin{array}{l}\text { Fabaceae/NX0265, } \\
\text { NX0691/W/H }\end{array}$ & Roots & Soaking & Oral & $\begin{array}{l}\text { Weakness/Tiredness General A04 } \\
\text { (85) }\end{array}$ \\
\hline Fen Ge & $\begin{array}{l}\text { Gai Gan } \\
\text { Er }\end{array}$ & $\begin{array}{l}\text { Pueraria montana var. } \\
\text { chinensis (Ohwi) } \\
\text { Sanjappa et Pradeep }\end{array}$ & $\begin{array}{l}\text { Fabaceae/ } \\
\text { NX0632/W/SC }\end{array}$ & $\begin{array}{l}\text { Roots, } \\
\text { flowers }\end{array}$ & Drying & Oral & $\begin{array}{l}\text { Elevated Blood Pressure K85 (86) } \\
\text { Headache N01 (103) } \\
\text { Neck Symptom L01 (74) } \\
\text { Pneumonia R81 (41) } \\
\text { Vertigo/Dizziness N17 (104) }\end{array}$ \\
\hline Mei & $\begin{array}{l}\text { Se Ka } \\
\text { Hao }\end{array}$ & $\begin{array}{l}\text { Prunus mume Siebold et } \\
\text { Zucc. }\end{array}$ & $\begin{array}{l}\text { Rosaceae/ } \\
\text { NX0435/C, W/T }\end{array}$ & Fruits & $\begin{array}{l}\text { Charring/ } \\
\text { drying }\end{array}$ & Topical/Oral & $\begin{array}{l}\text { Abdominal Pain D01 (104) } \\
\text { Asthma R96 (77) } \\
\text { Diarrhea D11 (86) } \\
\text { Nose Bleed/Epistaxis R06 (104) }\end{array}$ \\
\hline $\begin{array}{l}\text { Fen Zhi } \\
\text { Mei }\end{array}$ & Qi Pa Ke & $\begin{array}{l}\text { Rubus biflorus Buch.- } \\
\text { Ham. ex Sm. }\end{array}$ & $\begin{array}{l}\text { Rosaceae/NX0145, } \\
\text { NX0552/W/CS }\end{array}$ & $\begin{array}{l}\text { Roots, } \\
\text { branches, } \\
\text { leaves }\end{array}$ & Drying & Oral & $\begin{array}{l}\text { Menstruation Irregular/Frequent } \\
\text { X07 (67) }\end{array}$ \\
\hline $\begin{array}{l}\text { Mao Ye } \\
\text { Cha Tian } \\
\text { Pao }\end{array}$ & $\begin{array}{l}\text { Qi Dong } \\
\text { Bei }\end{array}$ & $\begin{array}{l}\text { Rubus coreanus var. } \\
\text { tomentosus Card. }\end{array}$ & $\begin{array}{l}\text { Rosaceae/ } \\
\text { NX0661/W/CS }\end{array}$ & Roots & Drying & Oral & $\begin{array}{l}\text { Cystitis/Urinary Infection other } \\
\text { U71 (100) } \\
\text { Menstruation Irregular/Frequent } \\
\text { X07 (67) } \\
\text { Prostate Symptom Y06 (53) } \\
\text { Urinary Calculus U95 (99) }\end{array}$ \\
\hline $\begin{array}{l}\text { Hong Pao } \\
\text { Ci Teng }\end{array}$ & $\begin{array}{l}\text { A He Le } \\
\text { De Ken }\end{array}$ & Rubus niveus Thunb. & $\begin{array}{l}\text { Rosaceae/NX0461, } \\
\text { NX0659/W/CS }\end{array}$ & $\begin{array}{l}\text { Roots, } \\
\text { leaves, } \\
\text { fruits }\end{array}$ & Drying & Oral & $\begin{array}{l}\text { Cystitis/Urinary Infection other } \\
\text { U71 (94)Menstruation Irregular/ } \\
\text { Frequent X07 (67) } \\
\text { Prostate Symptom Y06 (53) } \\
\text { Urinary Calculus U95 (99) }\end{array}$ \\
\hline $\begin{array}{l}\text { Zhou Zhi } \\
\text { Shu Li }\end{array}$ & $\begin{array}{l}\text { Qi Na } \\
\text { Ze }\end{array}$ & Rhamnus virgata Roxb. & $\begin{array}{l}\text { Rhamnaceae/ } \\
\text { NX0655/W/T }\end{array}$ & $\begin{array}{l}\text { Leaves, } \\
\text { branches }\end{array}$ & Drying & Oral & Malignancy A79 (86) \\
\hline $\mathrm{Da} \mathrm{Ma}$ & Sa & Cannabis sativa Linn. & $\begin{array}{l}\text { Cannabaceae/ } \\
\text { NX0561, NX0630/ } \\
\text { C,W/H }\end{array}$ & $\begin{array}{l}\text { Fruits, } \\
\text { leaves, } \\
\text { stem, bark }\end{array}$ & $\begin{array}{l}\text { Crushing/ } \\
\text { drying }\end{array}$ & Oral & Constipation D12 (46) \\
\hline $\begin{array}{l}\text { Rui Chi Hu } \\
\mathrm{Li}\end{array}$ & La Ze & $\begin{array}{l}\text { Quercus aliena var. } \\
\text { acutidentata Maxim. ex } \\
\text { Wenz. }\end{array}$ & $\begin{array}{l}\text { Fagaceae/ } \\
\text { NX0646/W/T }\end{array}$ & $\begin{array}{l}\text { Branches, } \\
\text { leaves }\end{array}$ & Drying & Oral & $\begin{array}{l}\text { Osteoarthrosis other L91 (101) } \\
\text { Rheumatoid/Seropositive } \\
\text { Arthritis L88 (104) }\end{array}$ \\
\hline $\begin{array}{l}\text { Chuan } \\
\text { Dian Gao } \\
\text { Shan Li }\end{array}$ & Bei Shi & $\begin{array}{l}\text { Quercus aquifolioides } \\
\text { Rehd. et Wils. }\end{array}$ & $\begin{array}{l}\text { Fagaceae/ } \\
\text { NX0241/W/T }\end{array}$ & $\begin{array}{l}\text { Fruits, bark, } \\
\text { flowers }\end{array}$ & Drying & Oral & $\begin{array}{l}\text { Nose Bleed/Epistaxis R06 (55) } \\
\text { Viral Hepatitis D72 (66) }\end{array}$ \\
\hline Hu Tao & $\begin{array}{l}\text { Gu Du } \\
\text { Bai Duo }\end{array}$ & Juglans regia Linn. & $\begin{array}{l}\text { Juglandaceae/ } \\
\text { NX0570/C, W/T }\end{array}$ & Bark & $\begin{array}{l}\text { Soaking/ } \\
\text { drying }\end{array}$ & Oral & $\begin{array}{l}\text { Cholecystitis/Cholelithiasis D98 } \\
\text { (63) } \\
\text { Diabetes Insulin Dependent T89 } \\
(75)\end{array}$ \\
\hline
\end{tabular}


Table 9 Ethnomedicinal data of medicinal plants and fungi recorded in the Dongba Sutras (Continued)

\begin{tabular}{|c|c|c|c|c|c|c|c|}
\hline $\begin{array}{l}\text { Chinese } \\
\text { name }\end{array}$ & $\begin{array}{l}\text { Naxi } \\
\text { name }\end{array}$ & Scientific name & $\begin{array}{l}\text { Family/voucher } \\
\text { specimen/ } \\
\text { habitat }^{\mathrm{a}} / \text { habit }^{\mathrm{b}}\end{array}$ & Part used & $\begin{array}{l}\text { Preparation } \\
\text { method }\end{array}$ & $\begin{array}{l}\text { Route of } \\
\text { administration }\end{array}$ & $\begin{array}{l}\text { Diseases treated/number of } \\
\text { respondents (ICPC-2) }\end{array}$ \\
\hline & & & & & & & $\begin{array}{l}\text { Diabetes Non-Insulin Dependent } \\
\text { T90 (69) } \\
\text { Dyspepsia/Indigestion D07 (90) } \\
\text { Influenza R80 (79) } \\
\text { Upper Respiratory Infection } \\
\text { Acute R74 (81) }\end{array}$ \\
\hline $\mathrm{Hu} \mathrm{Lu}$ & $\begin{array}{l}\text { Bei Pu } \\
\text { Gu De }\end{array}$ & $\begin{array}{l}\text { Lagenaria siceraria } \\
\text { (Molina) Standl. }\end{array}$ & $\begin{array}{l}\text { Cucurbitaceae/ } \\
\text { NX0675/C/HV }\end{array}$ & Leaves & Drying & Oral & $\begin{array}{l}\text { Genital Disease Male other Y99 } \\
\text { (68) }\end{array}$ \\
\hline Mao Gua & $\begin{array}{l}\text { Bu Luo } \\
\text { Lan }\end{array}$ & $\begin{array}{l}\text { Solena amplexicaulis } \\
\text { (Lam.) Gandhi }\end{array}$ & $\begin{array}{l}\text { Cucurbitaceae/ } \\
\text { NX0763/W/HV }\end{array}$ & Roots & Fresh & Topical & $\begin{array}{l}\text { Burn/Scald S14 (22) } \\
\text { Cough R05 (45) }\end{array}$ \\
\hline $\begin{array}{l}\text { Wu Bing } \\
\text { Jin Si Tao }\end{array}$ & $\begin{array}{l}\text { Ni Mei } \\
\text { Hei Tu } \\
\mathrm{Ba}\end{array}$ & $\begin{array}{l}\text { Hypericum augustini } \mathrm{N} . \\
\text { Robson }\end{array}$ & $\begin{array}{l}\text { Hypericaceae/ } \\
\text { NX0142/W/WV }\end{array}$ & $\begin{array}{l}\text { Whole } \\
\text { plants }\end{array}$ & $\begin{array}{l}\text { Crushing/ } \\
\text { drying }\end{array}$ & Oral/Topical & $\begin{array}{l}\text { Acute Hepatitis A D73 (75) } \\
\text { Dyspepsia/Indigestion D07 (48) } \\
\text { Genital Disease Male other Y99 } \\
\text { (90) } \\
\text { Gonorrhoea Male Y71 (101) } \\
\text { Pain General/Multiple Sites A01 } \\
\text { (12) } \\
\text { Prostate Symptom Y06 (96) } \\
\text { Psoriasis S91 (42) } \\
\text { Pyelonephritis/Pyelitis U70 (21) } \\
\text { Viral Hepatitis D72 (59)Worms/ } \\
\text { Other Parasites D96 (97) }\end{array}$ \\
\hline $\begin{array}{l}\text { Dian Nan } \\
\text { Shan Yang }\end{array}$ & La Ka & $\begin{array}{l}\text { Populus rotundifolia var. } \\
\text { bonatii (H. Lévl.) C. Wang } \\
\text { \& S. L. Tung }\end{array}$ & $\begin{array}{l}\text { Salicaceae/ } \\
\text { NX0672/W/T }\end{array}$ & Bark & $\begin{array}{l}\text { Soaking/ } \\
\text { drying }\end{array}$ & Oral/Topical & $\begin{array}{l}\text { Infectious Disease A78 (88) } \\
\text { Viral Disease A77 (79) } \\
\text { Worms/Other Parasites D96 (22) }\end{array}$ \\
\hline Chui Liu & Re Pei & Salix babylonica Linn. & $\begin{array}{l}\text { Salicaceae/ } \\
\text { NX0555/W/T }\end{array}$ & $\begin{array}{l}\text { Branches, } \\
\text { leaves, } \\
\text { roots }\end{array}$ & Fresh & Oral & Teeth/Gum Symptom D19 (17) \\
\hline $\begin{array}{l}\text { Qiu Hua } \\
\text { Liu }\end{array}$ & $\mathrm{Ji} \operatorname{Re}$ & Salix variegata Franch. & $\begin{array}{l}\text { Salicaceae/ } \\
\text { NX0563/W/T }\end{array}$ & $\begin{array}{l}\text { Branches, } \\
\text { leaves }\end{array}$ & Drying & Oral & $\begin{array}{l}\text { Haematuria U06 (97) } \\
\text { Urinary Calculus U95 (79) } \\
\text { Viral Hepatitis D72 (45) }\end{array}$ \\
\hline Zi Di Yu & $\begin{array}{l}\text { Qie Sai } \\
\text { Che E }\end{array}$ & $\begin{array}{l}\text { Geranium strictipes R. } \\
\text { Knuth }\end{array}$ & $\begin{array}{l}\text { Geraniaceae/ } \\
\text { NX0378/W/H }\end{array}$ & Roots & $\begin{array}{l}\text { Crushing/ } \\
\text { drying }\end{array}$ & Oral & $\begin{array}{l}\text { Dyspepsia/Indigestion D07 (82) } \\
\text { Mumps D71 (62) } \\
\text { Pneumonia R81 (75) } \\
\text { Viral Hepatitis D72 (48) }\end{array}$ \\
\hline $\begin{array}{l}\text { Yang Jiao } \\
\text { Tian Ma }\end{array}$ & $\begin{array}{l}\text { Ju Luo } \\
\text { Lan }\end{array}$ & $\begin{array}{l}\text { Dobinea delavayi (Baill.) } \\
\text { Baill. }\end{array}$ & $\begin{array}{l}\text { Anacardiaceae/ } \\
\text { NX0762/W/H }\end{array}$ & Roots & Crushing & Oral & $\begin{array}{l}\text { Limited Function/Disability (L) } \\
\text { L28 (53) }\end{array}$ \\
\hline $\begin{array}{l}\text { Chuan } \\
\text { Dian Wu } \\
\text { Huan Zi }\end{array}$ & Ba De Zi & $\begin{array}{l}\text { Sapindus delavayi } \\
\text { (Franch.) Radlk. }\end{array}$ & $\begin{array}{l}\text { Sapindaceae/ } \\
\text { NX0125/C, W/T }\end{array}$ & Fruits & Drying & Oral & Dyspepsia/Indigestion D07 (25) \\
\hline $\begin{array}{l}\text { Chuan } \\
\text { Lian }\end{array}$ & $\begin{array}{l}\text { Da Liu } \\
\text { Liu }\end{array}$ & $\begin{array}{l}\text { Melia toosendan Sieb. et } \\
\text { Zucc. }\end{array}$ & $\begin{array}{l}\text { Meliaceae/ } \\
\text { NX0169/W/T }\end{array}$ & $\begin{array}{l}\text { Whole } \\
\text { plants }\end{array}$ & Drying & Oral & $\begin{array}{l}\text { Abdominal Pain D01 (100) } \\
\text { Asthma R96 (94) } \\
\text { Diarrhoea D11 (96) }\end{array}$ \\
\hline Lang Du & $\begin{array}{l}\text { Lei Bu } \\
\text { Ne Du }\end{array}$ & $\begin{array}{l}\text { Stellera chamaejasme } \\
\text { Linn. }\end{array}$ & $\begin{array}{l}\text { Thymelaeaceae/ } \\
\text { NX0077/W/H }\end{array}$ & Roots & Crushing & Oral & Constipation D12 (85) \\
\hline $\begin{array}{l}\text { Lan Cang } \\
\text { Rao Hua }\end{array}$ & Wai De & Wikstroemia delavayi Lec. & $\begin{array}{l}\text { Thymelaeaceae/ } \\
\text { NX0066, NX0660/ } \\
\text { W/S }\end{array}$ & $\begin{array}{l}\text { Whole } \\
\text { plants, } \\
\text { flowers or } \\
\text { bark }\end{array}$ & Crushing & Oral & Epilepsy N88 (23) \\
\hline Wu Jing & A Ke & Brassica rapa Linn. & $\begin{array}{l}\text { Brassicaceae/ } \\
\text { NX0761/C/H }\end{array}$ & Roots & Drying & Oral & Bladder Symptom U13 (42) \\
\hline $\begin{array}{l}\text { Tong Qiao } \\
\text { She Gu }\end{array}$ & $\begin{array}{l}\mathrm{Mu} \mathrm{Gu} \\
\mathrm{Xu}\end{array}$ & $\begin{array}{l}\text { Balanophora involucrata } \\
\text { Hook. f. et Thomson }\end{array}$ & $\begin{array}{l}\text { Balanophoraceae/ } \\
\text { NX0502, NX0686/ } \\
\text { W/H }\end{array}$ & $\begin{array}{l}\text { Whole } \\
\text { plants }\end{array}$ & $\begin{array}{l}\text { Soaking/ } \\
\text { drying }\end{array}$ & Oral & $\begin{array}{l}\text { Neoplasm of Eye/Adnexa F74 } \\
(23) \\
\text { Orchitis/Epididymitis Y74 (85) } \\
\text { Trauma/Injury A80 (36) } \\
\text { Viral Hepatitis D72 (27) }\end{array}$ \\
\hline $\begin{array}{l}\text { San Chun } \\
\text { Shui Bai }\end{array}$ & Ji Xiu & $\begin{array}{l}\text { Myricaria paniculata P. Y. } \\
\text { Zhang et Y. J. Zhang }\end{array}$ & $\begin{array}{l}\text { Tamaricaceae/ } \\
\text { NX0197, NX0717/ }\end{array}$ & $\begin{array}{l}\text { Branches, } \\
\text { leaves }\end{array}$ & Drying & Ora & $\begin{array}{l}\text { Osteoarthrosis other L91 (103) } \\
\text { Rash Localized S06 (100) }\end{array}$ \\
\hline
\end{tabular}


Table 9 Ethnomedicinal data of medicinal plants and fungi recorded in the Dongba Sutras (Continued)

\begin{tabular}{|c|c|c|c|c|c|c|c|}
\hline $\begin{array}{l}\text { Chinese } \\
\text { name }\end{array}$ & $\begin{array}{l}\text { Naxi } \\
\text { name }\end{array}$ & Scientific name & $\begin{array}{l}\text { Family/voucher } \\
\text { specimen/ } \\
\text { habitat }^{\mathrm{a}} / \text { habit }^{\mathrm{b}}\end{array}$ & Part used & $\begin{array}{l}\text { Preparation } \\
\text { method }\end{array}$ & $\begin{array}{l}\text { Route of } \\
\text { administration }\end{array}$ & $\begin{array}{l}\text { Diseases treated/number of } \\
\text { respondents (ICPC-2) }\end{array}$ \\
\hline Zhi & & & W/S & & & & $\begin{array}{l}\text { Rheumatoid/Seropositive } \\
\text { Arthritis L88 (98) }\end{array}$ \\
\hline $\begin{array}{l}\text { Jin Qiao } \\
\text { Mai }\end{array}$ & $\begin{array}{l}\text { Ruo A } \\
\text { Kao Ken }\end{array}$ & $\begin{array}{l}\text { Fagopyrum dibotrys (D. } \\
\text { Don) Hara }\end{array}$ & $\begin{array}{l}\text { Polygonaceae/ } \\
\text { NX0490, NX0528/ } \\
\text { W/H }\end{array}$ & Roots & $\begin{array}{l}\text { Crushing/ } \\
\text { drying }\end{array}$ & Oral & $\begin{array}{l}\text { Abdominal Pain Epigastric D02 } \\
(69) \\
\text { Hair/Scalp Symptom S24 (65) } \\
\text { Heartburn D03 (78) } \\
\text { Mumps D71 (90) } \\
\text { Peptic Ulcer other D86 (49) }\end{array}$ \\
\hline $\begin{array}{l}\text { Huo Tan } \\
\mathrm{Mu}\end{array}$ & $\begin{array}{l}\text { Zei Lan } \\
\text { Xu }\end{array}$ & $\begin{array}{l}\text { Polygonum chinense } \\
\text { Linn. }\end{array}$ & $\begin{array}{l}\text { Polygonaceae/ } \\
\text { NX0708/C, W/H }\end{array}$ & $\begin{array}{l}\text { Whole } \\
\text { plants }\end{array}$ & Drying & Oral & $\begin{array}{l}\text { Cholecystitis/Cholelithiasis D98 } \\
\text { (61) }\end{array}$ \\
\hline $\begin{array}{l}\text { Dian Bian } \\
\text { Da Huang }\end{array}$ & $\begin{array}{l}\text { Lu Zei } \\
\text { Ken }\end{array}$ & Rheum delavayi Franch. & $\begin{array}{l}\text { Polygonaceae/ } \\
\text { NX0353/W/H }\end{array}$ & Roots & Drying/fresh & Oral/Topical & $\begin{array}{l}\text { Acute Bronchitis/Bronchiolitis } \\
\text { R78 (89) } \\
\text { Acute Hepatitis A D73 (103) } \\
\text { Bleeding/Haemorrhage NOS } \\
\text { A10 (26) } \\
\text { Chronic Bronchitis R79 (104) } \\
\text { Gastrointestinal Infection D70 } \\
\text { (71) } \\
\text { Haematuria U06 (59) } \\
\text { Heartburn D03 (99) } \\
\text { Influenza R80 (104) } \\
\text { Pneumonia R81 (104) } \\
\text { Upper Respiratory Infection } \\
\text { Acute R74 (97) }\end{array}$ \\
\hline $\begin{array}{l}\text { Li Jiang Da } \\
\text { Huang }\end{array}$ & $\begin{array}{l}\text { Ai San } \\
\text { Qi }\end{array}$ & Rheum likiangense Sam. & $\begin{array}{l}\text { Polygonaceae/ } \\
\text { NX0262, NX0693/ } \\
\text { W/H }\end{array}$ & Roots & $\begin{array}{l}\text { Soaking/ } \\
\text { drying }\end{array}$ & Oral & $\begin{array}{l}\text { Anal Fissure/Perianal Abscess } \\
\text { D95 (85) } \\
\text { Bleeding/Haemorrhage NOS } \\
\text { A10 (95) } \\
\text { Bursitis/Tendinitis/Synovitis NOS } \\
\text { L87 (73) } \\
\text { Gonorrhoea Female X71 (38) } \\
\text { Lump/Swelling Localized S04 } \\
\text { (85) } \\
\text { Melaena D15 (74) } \\
\text { Neck Symptom L01 (88) } \\
\text { Pain/Tenderness of Skin S01 (78) } \\
\text { Pain General/Multiple Sites A01 } \\
\text { (83) } \\
\text { Rectal Bleeding D16 (100) } \\
\text { Throat Symptom R21 (58) } \\
\text { Trauma/Injury A80 (79) } \\
\text { Viral Hepatitis D72 (79) }\end{array}$ \\
\hline $\begin{array}{l}\text { Yao Yong } \\
\text { Da Huang }\end{array}$ & $\begin{array}{l}\text { Hua Zei } \\
\text { De }\end{array}$ & Rheum officinale Baill. & $\begin{array}{l}\text { Polygonaceae/ } \\
\text { NX0753/C, W/H }\end{array}$ & Roots & Drying & Oral & $\begin{array}{l}\text { Constipation D12 (104) } \\
\text { Diarrhoea D11 (104) } \\
\text { Gonorrhoea Female X71 (104) }\end{array}$ \\
\hline $\begin{array}{l}\text { Ni Bo Er } \\
\text { Suan Mo }\end{array}$ & $\begin{array}{l}\text { Hua } \\
\text { Leng } \\
\text { Hua Zei } \\
\text { Ke }\end{array}$ & Rumex nepalensis Spreng. & $\begin{array}{l}\text { Polygonaceae/ } \\
\text { NX0074/W/H }\end{array}$ & Roots & Drying/fresh & Oral/Topical & $\begin{array}{l}\text { Constipation D12 (84) } \\
\text { Pruritus S02 (36) } \\
\text { Worms/Other Parasites D96 (90) }\end{array}$ \\
\hline Jin Tie Suo & $\begin{array}{l}\text { Du La } \\
\text { Pei }\end{array}$ & $\begin{array}{l}\text { Psammosilene tunicoides } \\
\text { W. C. Wu et C. Y. Wu }\end{array}$ & $\begin{array}{l}\text { Caryophyllaceae/ } \\
\text { NX0488/W/H }\end{array}$ & Roots & $\begin{array}{l}\text { Crushing/ } \\
\text { soaking }\end{array}$ & Topical & $\begin{array}{l}\text { Abdominal Pain Epigastric D02 } \\
\text { (35) } \\
\text { Bleeding/Hemorrhage NOS A10 } \\
\text { (103) } \\
\text { Musculoskeletal Disease other } \\
\text { L99 (104) } \\
\text { Osteoarthrosis other L91 (101) } \\
\text { Pain General/Multiple Sites A01 } \\
\text { (104) } \\
\text { Rheumatoid/Seropositive } \\
\text { Arthritis L88 (104) } \\
\text { Trauma/Injury A80 (98) }\end{array}$ \\
\hline Qian Sui & Mei Ru & Amaranthus & Amaranthaceae/ & Seeds & Drying & Oral & Dyspepsia/Indigestion D07 (39) \\
\hline
\end{tabular}


Table 9 Ethnomedicinal data of medicinal plants and fungi recorded in the Dongba Sutras (Continued)

\begin{tabular}{|c|c|c|c|c|c|c|c|}
\hline $\begin{array}{l}\text { Chinese } \\
\text { name }\end{array}$ & $\begin{array}{l}\text { Naxi } \\
\text { name }\end{array}$ & Scientific name & $\begin{array}{l}\text { Family/voucher } \\
\text { specimen/ } \\
\text { habitat }^{\mathrm{a}} / \text { habit }^{\mathrm{b}}\end{array}$ & Part used & $\begin{array}{l}\text { Preparation } \\
\text { method }\end{array}$ & $\begin{array}{l}\text { Route of } \\
\text { administration }\end{array}$ & $\begin{array}{l}\text { Diseases treated/number of } \\
\text { respondents (ICPC-2) }\end{array}$ \\
\hline$\overline{\mathrm{Gu}}$ & & hypochondriacus Linn. & NX0525/W/H & & & & Sleep Disturbance P06 (24) \\
\hline $\begin{array}{l}\text { Li Guo } \\
\text { Xian Ren } \\
\text { Zhang }\end{array}$ & $\begin{array}{l}\text { Cong } \\
\text { Hei }\end{array}$ & $\begin{array}{l}\text { Opuntia ficus-indica } \\
\text { (Linn.) Mill. }\end{array}$ & $\begin{array}{l}\text { Cactaceae/ } \\
\text { NX0109/C, W/H }\end{array}$ & $\begin{array}{l}\text { Whole } \\
\text { plants }\end{array}$ & Fresh & Topical & $\begin{array}{l}\text { Burn/Scald S14 (99) } \\
\text { Gonorrhoea Male Y71 (63) }\end{array}$ \\
\hline $\begin{array}{l}\text { Jun Qian } \\
\mathrm{Zi}\end{array}$ & Tao Zhi & Diospyros lotus Linn. & $\begin{array}{l}\text { Ebenaceae/ } \\
\text { NX0170/C, W/T }\end{array}$ & Fruits & Drying & Oral & Diarrhoea D11 (86) \\
\hline $\begin{array}{l}\text { Pu Tong } \\
\text { Lu Ti Cao }\end{array}$ & $\begin{array}{l}\text { Jiu Gu } \\
\text { Lei }\end{array}$ & Pyrola decorata $\mathrm{H}$. Andr. & $\begin{array}{l}\text { Ericaceae/NX0152, } \\
\text { NX0652/W/H }\end{array}$ & $\begin{array}{l}\text { Whole } \\
\text { plants }\end{array}$ & Drying & Oral & $\begin{array}{l}\text { Abdominal Pain Localized other } \\
\text { D06 (86) } \\
\text { Acute Bronchitis/Bronchiolitis } \\
\text { R78 (95) } \\
\text { Chronic Bronchitis R79 (79) } \\
\text { Influenza R80 (700) } \\
\text { Mouth/Tongue/Lip Symptom } \\
\text { D20 (79) } \\
\text { Upper Respiratory Infection } \\
\text { Acute R74 (69) }\end{array}$ \\
\hline $\begin{array}{l}\text { Ye Hua Du } \\
\text { Juan }\end{array}$ & $\begin{array}{l}\text { Shua } \\
\text { Dai Lan } \\
\text { Ba }\end{array}$ & $\begin{array}{l}\text { Rhododendron } \\
\text { racemosum Franch. }\end{array}$ & $\begin{array}{l}\text { Ericaceae/ } \\
\text { NX0085/W/S }\end{array}$ & $\begin{array}{l}\text { Branches, } \\
\text { flowers }\end{array}$ & Crushing & Topical & Psoriasis S91 (34) \\
\hline $\begin{array}{l}\text { Huang Bei } \\
\text { Du Juan }\end{array}$ & $\begin{array}{l}\text { Mu Gou } \\
\text { Ba Shi }\end{array}$ & $\begin{array}{l}\text { Rhododendron wardii W. } \\
\text { W. Sm. }\end{array}$ & $\begin{array}{l}\text { Ericaceae/NX0310, } \\
\text { NX0312/W/T }\end{array}$ & $\begin{array}{l}\text { Flowers, } \\
\text { fruits }\end{array}$ & $\begin{array}{l}\text { Crushing/ } \\
\text { drying }\end{array}$ & Oral/Topical & $\begin{array}{l}\text { Musculoskeletal Disease other } \\
\text { L99 (87) } \\
\text { Osteoarthrosis other L91 (76) } \\
\text { Rheumatoid/Seropositive } \\
\text { Arthritis L88 (82) }\end{array}$ \\
\hline $\begin{array}{l}\text { Dian Long } \\
\text { Dan Cao }\end{array}$ & $\mathrm{Ji} \mathrm{Ka}$ & $\begin{array}{l}\text { Gentiana rigescens } \\
\text { Franch. ex Hemsl. }\end{array}$ & $\begin{array}{l}\text { Gentianaceae/ } \\
\text { NX0350/W/H }\end{array}$ & $\begin{array}{l}\text { Whole } \\
\text { plants }\end{array}$ & $\begin{array}{l}\text { Crushing/ } \\
\text { soak }\end{array}$ & Oral & $\begin{array}{l}\text { Cholecystitis/Cholelithiasis D98 } \\
\text { (104) } \\
\text { Viral Hepatitis D72 (104) }\end{array}$ \\
\hline $\begin{array}{l}\text { Xi Nan Cu } \\
\text { Kang Shu }\end{array}$ & $\mathrm{Nu} \mathrm{Ao}$ & $\begin{array}{l}\text { Ehretia corylifolia C. H. } \\
\text { Wright }\end{array}$ & $\begin{array}{l}\text { Boraginaceae/ } \\
\text { NX0111/W/T }\end{array}$ & $\begin{array}{l}\text { Whole } \\
\text { plants }\end{array}$ & Soaking & Topical & Pruritus S02 (35) \\
\hline Ye Ba Zi & Ke Du & Elsholtzia rugulosa Hemsl. & $\begin{array}{l}\text { Lamiaceae/ } \\
\text { NX0178/W/H }\end{array}$ & $\begin{array}{l}\text { Leaves, } \\
\text { flowers }\end{array}$ & $\begin{array}{l}\text { Crushing/ } \\
\text { drying }\end{array}$ & Oral & $\begin{array}{l}\text { Influenza R80 (77) } \\
\text { Upper Respiratory Infection } \\
\text { Acute R74 (104) }\end{array}$ \\
\hline $\begin{array}{l}\text { Li Jiang } \\
\text { Huang Qin }\end{array}$ & $\begin{array}{l}\text { Bai Qi } \\
\text { Ba Pei } \\
\text { Ke }\end{array}$ & $\begin{array}{l}\text { Scutellaria likiangensis } \\
\text { Diels }\end{array}$ & $\begin{array}{l}\text { Lamiaceae/ } \\
\text { NX0696/W/H }\end{array}$ & Roots & Soak/drying & Oral/Buccal & Swallowing Problem D21 (95) \\
\hline $\begin{array}{l}\text { Bian Da } \\
\text { Xiu Qiu }\end{array}$ & $\begin{array}{l}\text { A You } \\
\text { Jian Da } \\
\text { Ke }\end{array}$ & $\begin{array}{l}\text { Hemiphragma } \\
\text { heterophyllum Wall. }\end{array}$ & $\begin{array}{l}\text { Plantaginaceae/ } \\
\text { NX0228/W/H }\end{array}$ & $\begin{array}{l}\text { Whole } \\
\text { plants }\end{array}$ & Drying & Oral & $\begin{array}{l}\text { Low Back Symptom L03 (75) } \\
\text { Menstruation Irregular/Frequent } \\
\text { X07 (33) } \\
\text { Musculoskeletal Disease other } \\
\text { L99 (69) } \\
\text { Osteoarthrosis other L91 (88) } \\
\text { Pain General/Multiple Sites A01 } \\
\text { (75) }\end{array}$ \\
\hline $\begin{array}{l}\text { Kuan Ye } \\
\text { Tu Er Feng }\end{array}$ & $\begin{array}{l}\text { Du Mei } \\
\text { Gu Fu } \\
\text { Pie }\end{array}$ & $\begin{array}{l}\text { Ainsliaea latifolia (D. } \\
\text { Don) Sch.-Bip. }\end{array}$ & $\begin{array}{l}\text { Asteraceae/ } \\
\text { NX0098/W/H }\end{array}$ & $\begin{array}{l}\text { Whole } \\
\text { plants }\end{array}$ & Drying & Oral & $\begin{array}{l}\text { Cough R05 (104) } \\
\text { Haemoptysis R24 (82) } \\
\text { Malaria A73 (36) } \\
\text { Rheumatoid/Seropositive } \\
\text { Arthritis L88 (87) }\end{array}$ \\
\hline $\begin{array}{l}\text { Niu Wei } \\
\text { HaO }\end{array}$ & Qi Ai & $\begin{array}{l}\text { Artemisia dubia Wall. ex } \\
\text { Bess. }\end{array}$ & $\begin{array}{l}\text { Asteraceae/ } \\
\text { NX0707/W/H }\end{array}$ & $\begin{array}{l}\text { Stem, } \\
\text { leaves }\end{array}$ & Soaking & Topical & $\begin{array}{l}\text { Menstruation Absent/Scanty X05 } \\
\text { (53) }\end{array}$ \\
\hline $\begin{array}{l}\text { Nan Ai } \\
\text { HaO }\end{array}$ & $\begin{array}{l}\text { Beng } \\
\text { Pei }\end{array}$ & $\begin{array}{l}\text { Artemisia verlotorum } \\
\text { Lam. }\end{array}$ & $\begin{array}{l}\text { Asteraceae/ } \\
\text { NX0358, NX0658/ } \\
\text { W/H }\end{array}$ & $\begin{array}{l}\text { Above- } \\
\text { ground } \\
\text { part }\end{array}$ & $\begin{array}{l}\text { Crushing/ } \\
\text { soaking/ } \\
\text { drying }\end{array}$ & Oral/Topical & $\begin{array}{l}\text { Anal Fissure/Perianal Abscess } \\
\text { D95 (45) } \\
\text { Influenza R80 (104) } \\
\text { Upper Respiratory Infection } \\
\text { Acute R74 (75) }\end{array}$ \\
\hline $\begin{array}{l}\text { Yun Nan } \\
\text { HaO }\end{array}$ & Beng Na & $\begin{array}{l}\text { Artemisia yunnanensis J. } \\
\text { F. Jeffrey ex Diels }\end{array}$ & $\begin{array}{l}\text { Asteraceae/ } \\
\text { NX0618/W/H }\end{array}$ & $\begin{array}{l}\text { Branches, } \\
\text { leaves }\end{array}$ & Crushing & Topical & Nose Bleed/Epistaxis R06 (68) \\
\hline Wu Jing & Ze Ge & Crepis napifera (Franch.) & Asteraceae/ & Roots & Fresh & Topical/Oral & Genital Disease Male other Y99 \\
\hline
\end{tabular}


Table 9 Ethnomedicinal data of medicinal plants and fungi recorded in the Dongba Sutras (Continued)

\begin{tabular}{|c|c|c|c|c|c|c|c|}
\hline $\begin{array}{l}\text { Chinese } \\
\text { name }\end{array}$ & $\begin{array}{l}\text { Naxi } \\
\text { name }\end{array}$ & Scientific name & $\begin{array}{l}\text { Family/voucher } \\
\text { specimen/ } \\
\text { habitat }^{\mathrm{a}} / \text { habit }^{\mathrm{b}}\end{array}$ & Part used & $\begin{array}{l}\text { Preparation } \\
\text { method }\end{array}$ & $\begin{array}{l}\text { Route of } \\
\text { administration }\end{array}$ & $\begin{array}{l}\text { Diseases treated/number of } \\
\text { respondents (ICPC- } 2 \text { ) }\end{array}$ \\
\hline $\begin{array}{l}\text { Huan } \\
\text { Yang Shen }\end{array}$ & & Babc. & NX0748/W/H & & & & $\begin{array}{l}\text { (46) } \\
\text { Visual Disturbance other F05 } \\
(25) \\
\text { Whooping Cough R71 (101) }\end{array}$ \\
\hline $\begin{array}{l}\text { Da Ding } \\
\text { Cao }\end{array}$ & $\begin{array}{l}\text { Jiu Ban } \\
\text { Er }\end{array}$ & $\begin{array}{l}\text { Gerbera anandria (Linn.) } \\
\text { Sch.-Bip. }\end{array}$ & $\begin{array}{l}\text { Asteraceae/ } \\
\text { NX0464/W/H }\end{array}$ & $\begin{array}{l}\text { Whole } \\
\text { plants }\end{array}$ & $\begin{array}{l}\text { Crushing/ } \\
\text { drying }\end{array}$ & Oral & $\begin{array}{l}\text { Gonorrhoea Female X71 (23) } \\
\text { Worms/Other Parasites D96 (52) }\end{array}$ \\
\hline Jie Gu Mu & $\begin{array}{l}\text { Su Kua } \\
\mathrm{Na}\end{array}$ & $\begin{array}{l}\text { Sambucus williamsii } \\
\text { Hance }\end{array}$ & $\begin{array}{l}\text { Adoxaceae/ } \\
\text { NX0049/C, W/S }\end{array}$ & Bark & Crushing & Topical & $\begin{array}{l}\text { Fracture: Femur L75 (95) } \\
\text { Fracture: Hand/Foot Bone L74 } \\
\text { (86)Fracture: Other L76 (78) } \\
\text { Fracture: Radius/Ulna L72 (77) } \\
\text { Fracture: Tibia/Fibula L73 (89) } \\
\text { Low Back Symptom L03 (63) } \\
\text { Osteoarthrosis other L91 (58) } \\
\text { Pain General/Multiple Sites A01 } \\
\text { (58) } \\
\text { Rheumatoid/Seropositive } \\
\text { Arthritis L88 (101) }\end{array}$ \\
\hline $\begin{array}{l}\text { Zhu Zi } \\
\text { Shen }\end{array}$ & $\begin{array}{l}\text { Man Hai } \\
\text { Lü }\end{array}$ & $\begin{array}{l}\text { Panax japonicus var. } \\
\text { major (Burkill) C. Y. Wu et } \\
\text { K. M. Feng }\end{array}$ & $\begin{array}{l}\text { Araliaceae/ } \\
\text { NX0536, NX0736/ } \\
\text { C,W/H }\end{array}$ & Roots & Crushing & Oral & $\begin{array}{l}\text { Elevated Blood Pressure K85 (96) } \\
\text { Genital Disease Male other Y99 } \\
\text { (29) } \\
\text { Low Back Symptom L03 (84) } \\
\text { Pain General/Multiple Sites A01 } \\
\text { (104) } \\
\text { Trauma/Injury A80 (104) }\end{array}$ \\
\hline $\begin{array}{l}\text { Chuan } \\
\text { Dian Chai } \\
\text { Hu }\end{array}$ & $\mathrm{Mu} \mathrm{Ru}$ & $\begin{array}{l}\text { Bupleurum candollei Wall. } \\
\text { ex DC. }\end{array}$ & $\begin{array}{l}\text { Apiaceae/ } \\
\text { NX0453/W/H }\end{array}$ & $\begin{array}{l}\text { Whole } \\
\text { plants }\end{array}$ & Drying & Oral & $\begin{array}{l}\text { Influenza R80 (100) } \\
\text { Pneumonia R81 (86) } \\
\text { Upper Respiratory Infection } \\
\text { Acute R74 (104) }\end{array}$ \\
\hline Hui Xiang & $\begin{array}{l}\text { Lai Wu } \\
\text { Ci E }\end{array}$ & Foeniculum vulgare Mill. & $\begin{array}{l}\text { Apiaceae/ } \\
\text { NX0108/C/H }\end{array}$ & $\begin{array}{l}\text { Whole } \\
\text { plants }\end{array}$ & Fresh & Oral & $\begin{array}{l}\text { Abdominal Pain Localized other } \\
\text { D06 (46) } \\
\text { Bedwetting/Enuresis P12 (24) } \\
\text { Cystitis/Urinary Infection other } \\
\text { U71 (77)Gonorrhoea Female X71 } \\
\text { (23) } \\
\text { Orchitis/Epididymitis Y74 (31) } \\
\text { Urinary Frequency/Urgency U02 } \\
\text { (101) }\end{array}$ \\
\hline $\begin{array}{l}\text { Bai Liang } \\
\text { Du Huo }\end{array}$ & $\begin{array}{l}\text { Guo Ru } \\
\text { Ke }\end{array}$ & $\begin{array}{l}\text { Heracleum candicans } \\
\text { Wall. ex DC. }\end{array}$ & $\begin{array}{l}\text { Apiaceae/ } \\
\text { NX0334/W/H }\end{array}$ & Roots & $\begin{array}{l}\text { Crushing/ } \\
\text { drying }\end{array}$ & Oral & $\begin{array}{l}\text { Abdominal Pain D01 (86) } \\
\text { Abdominal Pain Epigastric D02 } \\
\text { (69) } \\
\text { Cough R05 (58) }\end{array}$ \\
\hline
\end{tabular}

Angiosperms are sorted follows APG IV

a Habitat: W, wild; C, cultivated

bHabit: H: herbs; T: trees; S: shrubs; WV: woody vines; CS: climbing shrubs; HV: herbal vines; M: Macrofungi

small district, and Reineckea carnea and Hypericum augustinii are endemic to China. This emphasizes the uniqueness of Naxi medicinal plants.

\section{Analysis of endemic species}

Among the medicinal plants in the Dongba Sutras, 25 species are endemic to China, accounting for $29.41 \%$ of the total number of medicinal plant species in the Dongba Sutras (85 species) (Table 10). Moreover, there are eight species only distributed in the areas inhabited by Naxi people (Fig. 2), including northwest Yunnan, southwest Sichuan, and Southeast Tibet. Examples include Populus rotundifolia var. bonatii, Rheum likiangense, Chesneya polystichoides, Geranium strictipes, Dobinea delavayi, Wikstroemia delavayi, Rhododendron wardii, and Scutellaria likiangensis.

The Naxi people consider human beings and nature as brothers. This ecological ethics concept lays the foundation for the Naxi people to live in harmony with nature; it shows the most primitive and simple concept of environmental conservation by human beings [30]. The distribution area of these plant species is very small. Although the Naxi people have been using these plants as medicinal materials for a long time, their populations are still stable, indicating that Naxi people attach great importance to plant conservation when collecting these 
Table $\mathbf{1 0}$ Chinese endemic plant species recorded in the Dongba Sutras

\begin{tabular}{|c|c|c|c|c|c|}
\hline ID & Family & Scientific name & Distribution* & Abundance ${ }^{* *}$ & Resource type \\
\hline 1 & Anacardiaceae & Dobinea delavayi & SW & Sol & Wild \\
\hline 2 & Boraginaceae & Ehretia corylifolia & SW & Cop1 & Wild \\
\hline 3 & Caryophyllaceae & Psammosilene tunicoides & SW & Sp & Wild \\
\hline 4 & Compositae & Artemisia yunnanensis & SW,W & Cop2 & Wild \\
\hline 5 & Compositae & Crepis napifera & SW & Cop2 & Wild \\
\hline 6 & Cupressaceae & Cupressus duclouxiana & SW & Cop1 & Wild \\
\hline 7 & Ericaceae & Rhododendron racemosum & SW & Cop3 & Wild \\
\hline 8 & Ericaceae & Rhododendron wardii & sw & Cop1 & Wild \\
\hline 9 & Geraniaceae & Geranium strictipes & sw & Cop1 & Wild \\
\hline 10 & Gramineae & Fargesia orbiculata & SW & Cop1 & Wild \\
\hline 11 & Guttiferae & Hypericum augustinii & SW & Cop3 & Wild \\
\hline 12 & Labiatae & Scutellaria likiangensis & SW & Cop1 & Wild \\
\hline 13 & Leguminosae & Chesneya polystichoides & sw & Sp & Wild \\
\hline 14 & Liliaceae & Asparagus meioclados & SW & Cop1 & Wild \\
\hline 15 & Magnoliaceae & Magnolia delavayi & SW & Sp & Cultivated \\
\hline 16 & Pinaceae & Pinus yunnanensis & $S W, S$ & Soe & Wild \\
\hline 17 & Polygonaceae & Rheum likiangense & sw & Sp & Wild \\
\hline 18 & Polygonaceae & Rheum officinale & $S W, S, C$ & Cop2 & Cultivated,wild \\
\hline 19 & Rosaceae & Rubus coreanus var. tomentosus & SW,C,W & Cop2 & Wild \\
\hline 20 & Sabiaceae & Meliosma cuneifolia & $S W, C, W$ & Cop1 & Wild \\
\hline 21 & Salicaceae & Populus rotundifolia var. bonatii & sw & Soe & Wild \\
\hline 22 & Salicaceae & Salix variegata & $S W, C, W$ & Cop3 & Wild \\
\hline 23 & Sapindaceae & Sapindus delavayi & SW,C & Sp & Cultivated,wild \\
\hline 24 & Tamaricaceae & Myricaria paniculata & $S W, C, W$ & Cop1 & Wild \\
\hline 25 & Thymelaeaceae & Wikstroemia delavayi & SW & Cop1 & Wild \\
\hline
\end{tabular}

*Note:SW-Southwest China; C-Central China; W-West China; S-South China

**Soe (Sociales): High number of individuals, the above-ground plant part is closed

Cop3 (Copiosae): High number of individuals, but the above-ground plant part is not closed

Cop2: Large and common plants

Cop1: Large plants, but small populations

Sp (Sparsal): Low number of plants, scattered

Sol (Solitariae): Low number of plants, sparse

Un (Unicum): Only one individual

medicinal plants. The Naxi people collect medicinal materials from their surroundings to treat many diseases. They never harm the environment during plant collecting, and they are grateful for being able to take advantage of wild medicinal plants. This fully embodies their idea of maintaining ecological balance. Meanwhile, artificial cultivation was adapted to expand the population of medicinal plants with rare natural resources in order to minimize their impact on wild plant resources.

\section{Conclusions}

\section{A variety of herbal medicine was recorded in the Dongba sutras}

The medicinal plants used by the Naxi people are diverse. A variety of herbal medicine closely related to the life of the Naxi people was recorded in the Dongba Sutras. A total of
85 species of medicinal plants and fungi belonging to 51 families and 71 genera were recorded in the Dongba Sutras, among which 25 species are endemic to China, and 8 species are distributed in a small region. There were 22 species of medicinal dietary plants recorded in the Dongba Sutras.

\section{The basic features of traditional Naxi medicine}

The knowledge of traditional Naxi medicine is always in the hands of the elderly and clergy. The traditional apprenticeship between the elderly and the young makes an assurance of the knowledge inheritance from age to age. Dongba, as the clergyman in the Naxi people, records the most important medical knowledge in the Dongba Sutras for better inheritance. 

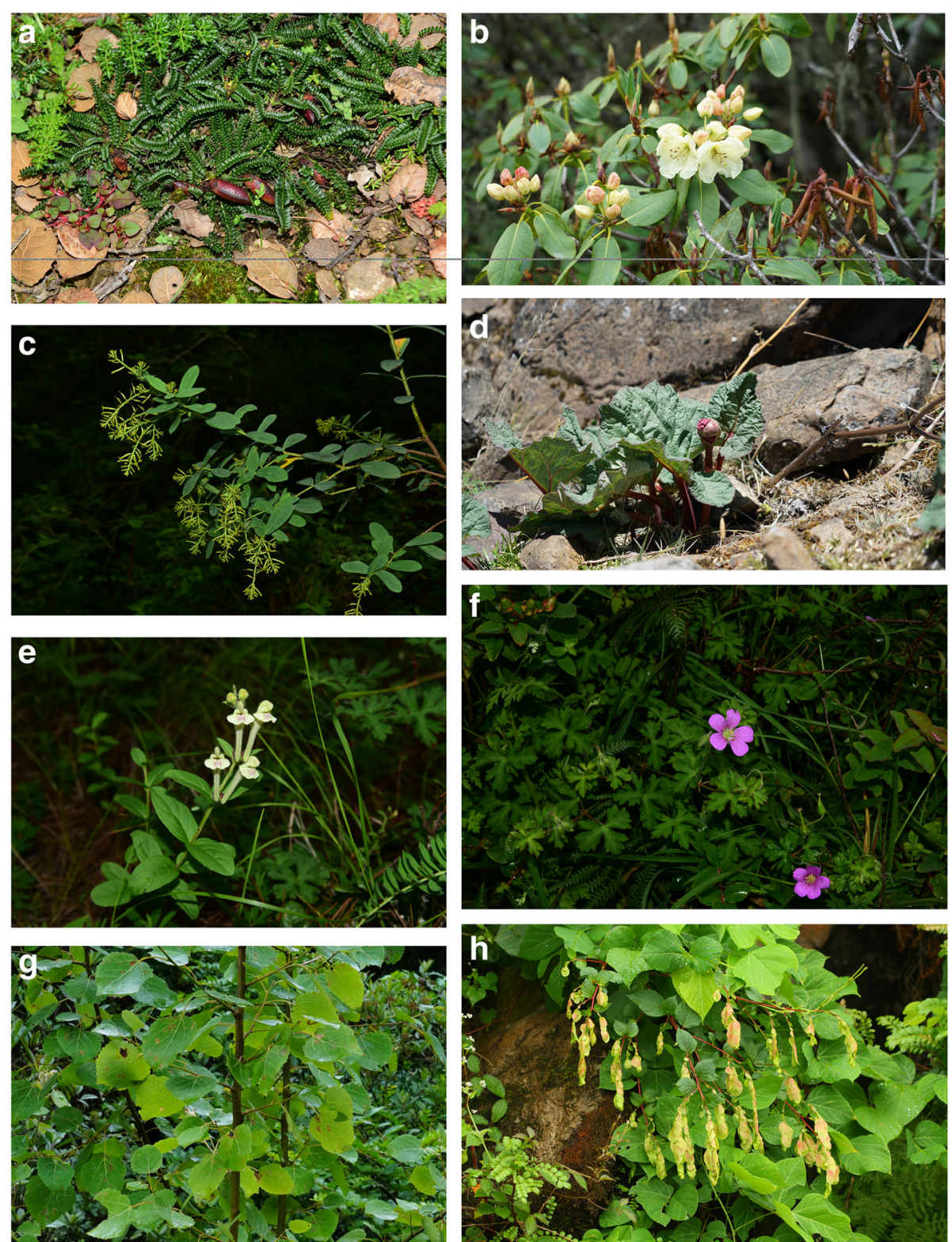

Fig. 2 Eight plant species endemic to China present in the areas inhabited by Naxi people. a: Chesneya polystichoides; $\mathbf{b}$ : Rhododendron wardil; $\mathbf{c}$ Wikstroemia delavayi; d: Rheum likiangense; e: Scutellaria likiangensis; $\mathbf{f}$ : Geranium strictipes; $\mathbf{g}$ : Populus rotundifolia var. bonatii; $\mathbf{h}$ : Dobinea delavayi

In the processing of medicinal materials, Naxi people make good use of fresh products, medicinal liquids, and plant powders. No complex processing is required from the raw plants to the medicine used, which is very convenient. Medicinal liquids can fully dissolve alcohol-soluble active substances and are easy to store. Different types of mixed powder are used internally or externally suiting the remedy to the different cases, which not only brings convenience to clinical uses but also protects the intellectual property rights of the folk healers because it is hard to know which medicinal plants are used in the powders.

The Naxi ancestors inhabit mountainous areas and are seldom influenced by alien cultures. As a result, the methods of medication are easy to follow, mainly including decocting, oral consumption with warm water, and topical. And the processing technology of Naxi medicine only includes some simple procedures like washing, drying, and crushing.

Four groups of diseases are common diseases in Naxi people living areas: they are the group of digestive 


\begin{tabular}{|c|c|c|c|c|c|c|}
\hline Scientific name & Scientific name & hieroglyphs & Scientific name & hieroglyphs & Scientific name & hieroglyphs \\
\hline Engleromyces goetzi & Schizophyllum commune & 出 $a=0$ m & Dobinea delavayi & है है B: & Sapindus delavayi का mo & ma \\
\hline Poria cocos & Selaginella pulvinata & 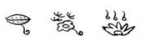 & Melia toosendan & \& & Stellera chamaejasme & 起㱠的 \\
\hline Equisetum ramosissimum $\neq 2 S$ & Drynaria delavayi & 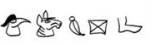 & Wikstroemia delavayi & $\infty x$ & Brassica rapa & का \\
\hline Picea likiangensis & Pinus armandii & 半 & Balamophora involucrata & 多篎丛 & Myricaria paniculata & $\$$ \\
\hline Pinus yunnanensis & Cupressus duclouxiama & $\$ 3$ & Fagopyrum dibotrys & कृ & Polygonum chinense & $\rho$ 照总 \\
\hline Juniperus squamata & Schisandra rubriflora & P舟远 & Rheum delavayi & $\leftrightarrow \equiv \infty$ & Rheum likiangense & ఠ庭必 \\
\hline Magnolia delavayi & Neocinnamomum delavayi & 近? & Rheum officinale & 鸪 $\curvearrowleft$ & Rumex nepalensis & $\curvearrowright$ 果 \\
\hline Acorus calamus & Alisma orientale & $\pi 0$ & Psammosilene tunicoides & DLEs & Amaranthus hypochondriacus & $s \quad \$$ \\
\hline Gymnadenia conopsea & Gymnadenia orchidis & 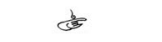 & Opuntia ficus-indica & 触又 & Diospyros lotus & 会暃 \\
\hline Spiranthes sinensis & Allium ascalonicum & 定 & Pyrola decorata & 头等熋 & Rhododendron racemosum & 上 $\mathbb{V}$ \\
\hline Allium hookeri & Allium sativum & 热 & Rhododendron wardii & of & Gentiana rigescens & 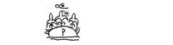 \\
\hline Asparagus meioclados & Reineckea carnea & $\leadsto \square \propto \propto ⿻$ & Ehretia corylifolia & 萧 & Elsholtzia rugulosa & 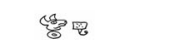 \\
\hline Hedychitumyunnanense & Fargesia orbiculata & 美 & Scutellaria likiamgensis & er & Hemiphragma heterophyllum & 答哭䟚 \\
\hline Setaria italica var. germanica & Clematis chrysocoma & 国来平 & Ainslinea latifolia & 崖回 & Artemisia dubia & हि \\
\hline Clematis connata & Meliosma cuneifolia & 甍 & Artemisia verlotorum & わ艮り & Artemisia yunnanensis & $3^{33^{3}}$ \\
\hline Chesneya polystichoides & Pueraria montana var. chinens & sis $\pi \Delta \longleftrightarrow$ & Crepis napifera & $\theta \sqrt{8}$ & Gerbera anandria & $\frac{\pi}{8}$ s \\
\hline Prunus mume & Rubus biflorus & 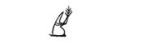 & Sambucus williamsii & 厽宋嘌 & Panax japonicus var. major & $\frac{6}{3} \cos \frac{\pi}{4}$ \\
\hline Rubus coreanus var. tomentosus & Rubus niveus & 类 & Bupleurum candollei & 番 & Foeniculum vulgare & ब局以 \\
\hline Rhamnus virgata & Cannabis sativa & 䩣 & Solena amplexicaulis & (o) 酸 & Hypericum augustini & की \\
\hline Quercus aliena var. acutidentata & Quercus aquifolioides & 诺 & Populus rotundifolia var. bonutii & $i=e^{2}$ & Salix babylonica & $\psi$ \\
\hline 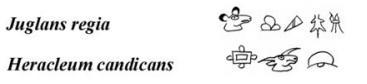 & Lagenaria siceraria & 的当 & Salix variegata & 幽 & Geranium strictipes & 厄静突 \\
\hline
\end{tabular}

diseases (D), followed by the respiratory (R), musculoskeletal (L), and the general and unspecified disease group (A). The Naxi folk healers have a high consensus on the treatment of these diseases.

\section{The ecological ethics of Naxi people have positive significance for the conservation of wild plant resources}

Hengduan mountainous where the Naxi people who live own one of the greatest abundant biodiversities in the world. Naxi people always keep the scientific ecological ethics concept in mind. The Naxi people never harm the environment during plant collecting, and they are grateful for being able to take advantage of wild medicinal plants. Meanwhile, artificial cultivation is adapted to expand the population of medicinal plants with rare natural resources in order to minimize their impact on wild plant resources.

Dongba Sutras are recorded in hieroglyphics (Fig. $3)$; thus, only the Dongbas, as the clergymen, can fully understand them. Contents of the Dongba Sutras are all-encompassing. Medical knowledge only takes a small part of the whole contents, and the records are not comprehensive enough. In addition, the folk medicinal knowledge is orally passed down. Thus, it is necessary to further deepen the investigation and research efforts to systematically organize and catalog the Naxi people's unique traditional medicine, exhibiting its due brilliance.

\section{Abbreviations \\ APG IV: The Angiosperm Phylogeny Group classification for the orders and families of flowering plants ed.IV; AQSIQ: General Administration of Quality Supervision of China; IMDY: The Herbarium, Yunnan Branch, Institute of Medicinal Plants, Chinese Academy of Medical Science; ICF: The informant consensus factor; ICPC-2: International Classification of Primary Care, revised second ed; WONCA: World Organization of Family Doctors; WHO: World Health Organization}

\section{Acknowledgements}

We are grateful to Yunnan Provincial Office of the Fourth National Census of Traditional Chinese Medicine Resources and Lijiang Municipal Government, all members of Lijiang Medical Association of Minorities, and Academician Luqi Huang, President of Chinese Academy of Traditional Chinese Medicine for their help during the study.

\section{Authors' contributions}

Jianqin Liu designed the study. Jingyuan Song designed and revised the manuscript. Haitao Li performed the ethnobotanical plant surveys; prepared the herbarium samples, botanical identification of the plant species, data curation data, formal analysis, analysis, and writing - original draft; and wrote the manuscript. Zhiyong Li performed the ethnobotanical plant surveys, data curation data, and formal analysis. Xiaobo Zhang contributed to the data curation data and formal analysis. Shaohua Yang performed the ethnobotanical plant surveys and prepared the herbarium samples and botanical identification of plant species. Cui Chen performed the ethnobotanical plant surveys and prepared herbarium samples and botanical identification of plant species. Qingning Yang performed the ethnobotanical plant surveys and prepared herbarium samples and data curation.

Chengfeng He performed the ethnobotanical plant surveys and prepared the herbarium samples and data curation. The authors have read and approved the final version of the manuscript. 


\section{Funding}

This research was supported by the accreditation scheme from the State Administration of Traditional Chinese Medicine of the People's Republic of China (Grant No. GZY-KJS-2018-004), Public Health Service Subsidy of Traditional Chinese Medicine in 2018 "The Project of National Census of Traditional Chinese Medicine Resources" ((2018) No. 43), and Yunnan provincial Science and Technology Major Projects(202002AA100007).

\section{Availability of data and materials}

All data generated or analyzed during this study are included in this published article (and its supplementary information files).

\section{Declarations}

Ethics approval and consent to participate

Not applicable.

\section{Consent for publication}

Prior and informed consent of local people's pictures had been obtained for publication.

\section{Competing interests}

The authors declare that they have no competing interests.

\section{Author details}

${ }^{1}$ Key Lab of Chinese Medicine Resources Conservation, State Administration of Traditional Chinese Medicine of the People's Republic of China, Institute of Medicinal Plant Development, Chinese Academy of Medical Sciences \& Peking Union Medical College, Beijing 100193, People's Republic of China. ${ }^{2}$ Yunnan Key Laboratory of Southern Medicinal Utilization, Yunnan Branch, Institute of Medicinal Plant Development, Chinese Academy of Medical Sciences \& Peking Union Medical College, Jinghong 666100, People's Republic of China. ${ }^{3}$ School of Pharmacy, Minzu University of China, Beijing 100081, People's Republic of China. ${ }^{4}$ Yunnan Province Resources of Development and Collaborative Innovation Center for New Traditional Chinese Medicine, Kunming 650051, People's Republic of China. ${ }^{5}$ State Key Laboratory Breeding Base of Dao-di Herbs, National Resource Center for Chinese Materia Medical, China Academy of Chinese Medical Sciences, Beijing 100700, People's Republic of China. ${ }^{6}$ Institute of Alpine Economics and Botany, Yunnan Academy of Agricultural Sciences, Lijiang 674100, People's Republic of China. ${ }^{7}$ Lijiang Medical Association of Minorities, Lijiang 674100, People's Republic of China.

\section{Received: 4 February 2021 Accepted: 15 April 2021}

\section{Published online: 29 April 2021}

\section{References}

1. Wang $Y$, Zheng J. A flower of national medicine nurtured by Dongba culture. Journal of Yunnan University of Traditional Chinese Medicine. 2006; 4:55. https://doi.org/https://doi.org/10.19288/j.cnki.issn.1000-2723.2006.04. 023.

2. Rock, J. F.. The Ancient Na-Khi Kingdom of Southwest China. Cambridge,MA: Harvard University Press; 1947, DOl: https://doi.org/10.4159/harvard. 9780674289192.

3. Chen HG. Dongba Sutras: an Encyclopedia of ancient Naxi Society. Yunnan Archives. 1999; 5:26.

4. Chen HY. Research on the excavation and utilization of ancient medical books of ethnic minorities in Southwest China. Beijing: Nationalities Publishing House; 2011.

5. Chen YX, Xu LY, Yang LF. Research on excavation and utilization of Naxi Dongba ancient medical literature from the perspective of national memory inheritance. Chinese J Ethnomed Ethnopharm. 2018;27(14):10-3.

6. Institute of Dongba Culture. The complete works of Dongba sutras in Naxi. Kunming: Yunnan People's Publishing House; 1999.

7. Lijiang Municipal People's Government Portal, 2020. http://www.lijiang.gov. cn/culture/. Accessed 23 October 2020.

8. Yunnan Network, 2020. http://lijiang.yunnan.cn/system/2018/07/03/03 0014212.shtml. Accessed 23 October 2020

9. China Weather Network. Lijang City, Analysis of Lijiang Climate Background for the period of 1971-2000. 2020. http://www.weather.com.cn/cityintro/1 01291401.shtml. Accessed 23 October 2020.
10. Martin GJ. Ethnobotany: a methods manual, WWF for nature international. London: Chapman and Hall; 1995. https://doi.org/10.1007/978-1-4615-2496-0.

11. International Society of Ethnobiology. ISE code of ethics (with 2008 additions). 2006.

12. Ministry of Education of the People's Republic of China website. 1958. http://www.moe.gov.cn/jyb_sjzl/ziliao/A19/195802/t19580201_186000.html. Accessed 23 October 2020.

13. AQSIQ, SAC. Gb/T16159. Basic Rules for Hanyu Pinyin Orthography. 2012.

14. International Classification Committee of Wonca. International classification of primary care, revised second ed. Oxford: Oxford University Press; 2015.

15. Yebouk C., Redouan F.Z., Benítez G., Bouhbal M., Kadiri M., Boumediana A.l., Molero-Mesa J., Merzouki A. Ethnobotanical study of medicinal plants in the Adrar Province, Mauritania J Ethnopharmacol 2020; 246: 112217. https://doi. org/https://doi.org/10.1016/j.jep.2019.112217.

16. Staub P.O., Geck M.S., Weckerle C.S., Casu L., Leonti M. Classifying diseases and remedies in ethnomedicine and ethnopharmacology. J Ethnopharmacol. 2015; 174:514-519. https://doi.org/https://doi.org/10.1016/j.jep.2015.08.051.

17. Editorial Committee of Flora of China. Flora of China. Beijing: Science Press; 1989-2013.

18. Editorial Committee of Flora Reipublicae Popularis Sinicae. Flora Peipublicae Popularis Sinicae. Beijing: Science Press; 1959-2004.

19. Editorial Committee of Flora Yunnanica. Flora Yunnanica. Beijing: Science Press; 1977-2006.

20. The Plant List. 2020. http://www.theplantlist.org. Accessed 23 October 2020.

21. The Angiosperm Phylogeny Group, M. W. Chase, M. J. M. Christenhusz, M. F. Fay, J. W. Byng, W. S. Judd, D. E. Soltis, D. J. Mabberley, A. N. Sennikov, P. S. Soltis, P. F. Stevens. An update of the angiosperm phylogeny group classification for the orders and families of flowering plants: APG IV, Bot J Linn Soc 2016;181(5):1-20. https://doi.org/https://doi.org/10.1111/boj.12385.

22. Wang $\mathrm{YH}$, Wang C. Common research methods of ethnobotany. Hangzhou: Zhejiang Education Publishing House; 2017.

23. Chinese Pharmacopoeia Commission. Pharmacopoeia of the People's republic of China. Beijing: China Medicinal Science Press; 2020.

24. Wu GF, Feng ZJ, Ma WL, Zhou XJ, Lang KC, Hu RL, Wang CJ, Li RG. Botany. Beijing: Higher Education Press; 1992. p. 342

25. Ministry of Ecology and Environment of the People's Republic of China website. 2014. http://www.mee.gov.cn/ywgz/fgbz/bz/bzwb/stzl/201411/t2 0141106_291239.shtml. Accessed 23 October 2020.

26. Xie GZ, Tang XY, Li XJ, Liu H, Zhang SH. Development and application of "one root of medicine and food", modern Chin Med 2020;22(09):1428-1433. https://doi.org/https://doi.org/10.13313/j.issn.1673-4890.20200229001

27. Zhang, L., Zhang, Y., Pei, S., Geng Y., Wang C., Yuhua W. Ethnobotanical survey of medicinal dietary plants used by the Naxi people in Lijiang area, Northwest Yunnan, China. J ethnobiology Ethnomedicine. 2015; 11:40. https://doi.org/https://doi.org/10.1186/s13002-015-0030-6, 1.

28. Dalar A., Mukemre M., Unal M., Ozgokce F. Traditional medicinal plants of Ağrı Province, Turkey. J Ethnopharmacol. 2018;226:56-72. https://doi.org/ https://doi.org/10.1016/j.jep.2018.08.004

29. Li GY, Cheng YG, Zeng TC, Li MJ, Sun RR, Li HF, Kong XP, Pei MR. Action mechanism of total flavonoids of Diaphragma Juglandis Fructus in treating type 2 diabetes mellitus based on network pharmacology and cellular experimental validation of AKT/FoxO1 signaling pathway. Drug Evaluation Research 2019;42(1): 30-40. https://doi.org/https://doi.org/10.7501/j.issn.2019.01.005.

30. Zheng J, Wang Y. Study on Dongba medicine of Naxi nationality. Kunming: Yunnan Science and Technology Press; 2006.

\section{Publisher's Note}

Springer Nature remains neutral with regard to jurisdictional claims in published maps and institutional affiliations. 